This item was submitted to Loughborough's Research Repository by the author.

Items in Figshare are protected by copyright, with all rights reserved, unless otherwise indicated.

\title{
Nonlinear internal waves in the upper atmosphere
}

PLEASE CITE THE PUBLISHED VERSION

LICENCE

CC BY-NC-ND 4.0

\section{REPOSITORY RECORD}

Caillol, P.. 2019. "Nonlinear Internal Waves in the Upper Atmosphere". figshare. https://hdl.handle.net/2134/382. 


\title{
Nonlinear internal waves in the upper atmosphere
}

\author{
P. CAILLOL ${ }^{1}$ \\ Department of Mathematical Sciences, Loughborough University, Loughborough, LE11 3TU, UK
}

\begin{abstract}
This paper considers the large-scale dynamics generated in the upper atmosphere by the destabilization of a linear internal gravity wave and its eventual restabilization through nonlinear processes into a coherent pattern. The assumption of a strongly dissipative medium is relevant to wave propagation in the thermosphere range above $100 \mathrm{~km}$. A parametric instability analysis is carried out involving the Froude, Reynolds and Prandtl numbers, and the following parameters: friction, wave incidence and disturbance periodicity. A solution of the motion is sought with the help of a multiscale technique based upon the hypothesis of large-scale flow. The leading-order amplitude equation governing this dynamics contains the Cahn-Hilliard equation, but possesses, as well, a dispersive term depending on the Prandtl number, a large-scale damping proportional to the overall Richardson number and a quadratic term modelling the incidence effects. A numerical and analytical study of this equation is given. An internal-wave stability criterion is obtained which shows the possibility of obtaining coherent structures for large Froude numbers.
\end{abstract}

Keywords: Internal Wave; Viscous and Stratified Shear Flow Instability; Prandtl Number

\section{Introduction}

The theory of stability of viscous and stratified shear flows and their transition to turbulence has been subject to extensive study for several decades. Numerous applications in geophysical and astrophysical motions motivate this research. The interaction of large-scale vortices and waves with small-scale turbulence characterizes such flows.

We consider here the instability of a small-vertical-scale internal gravity wave in a density-stratified and dissipative medium. This linear wave propagates nearly vertically in a vertical plane. The analysis of the stability of such a wave is of interest in the context of the propagation of internal waves in the upper atmosphere. Observational data indicate that short internal waves propagate with a wavenumber very close to the vertical direction (Meek, Reid \& Manson (1985)). The study of an internal wave moving in a dissipative medium restricts its area of application to the thermosphere. Molecular viscosity and thermal conduction inhibit the upward propagation of gravity waves. With increasing height, only large-scale waves can survive this attenuation (Kato (1980)). Numerous studies of linear stability in inviscid fluids show that these waves are unstable whatever the wave amplitude and for a large wavenumber range (Drazin (1977); Kurganskiy (1980)). The present work investigates two-dimensional wave instabilities taking into account both large and small-scale dissipations. The interest is twofold: first, the purpose is to examine the effects of the fluid parameters like the Prandtl number and the large-scale friction on the wave stability at a relatively low Reynolds number, because even though these effects have already been considered in experiments, (Thorpe (1994)), they have not been studied theoretically yet. Secondly, while internal waves are known to break down in the mid-troposphere and in the stratosphere (Lombard and Riley (1996)), we would like to demonstrate the possible existence of a long-lived and long-wavelength nonlinear wave in the thermosphere. This wave would be generated by the destabilization of a linear internal wave and by its restabilization. The stability analysis will give the relevant régime for which we will observe a threshold of instability characterized by Reynolds and Péclet number minima located at a finite but small disturbance wavenumber. We will show that the inducing instability is then dynamical. An asymptotic analysis valid in the neighbourhood of this threshold will enable us to investigate the evolution of the instability through numerical and analytical means. Previous works showed the possibility of the emergence of coherent patterns in an unstable stratified shear flow. Batchayev and Kurganskiy (1986) succeeded in obtaining experimentally a stationary régime constituted by a succession of eddies created from the destabilization of such a flow. Balmforth and Young (2002) analysed the instability threshold of this motion theoretically and numerically for a large range of Péclet and Reynolds numbers; however their approach only permitted very small Richardson numbers. When stratification is weak, the marginal stability possesses a minimum located at a very small wavenumber. For stronger stratification,

\footnotetext{
${ }^{1}$ Corresponding author E-mail: caillol@math.mcgill.ca
} 
instability appears at smaller scales (Kurganskiy (1980); Batchayev and Kurganskiy (1986)). A weakly stratified fluid is therefore assumed in order to obtain large-scale structures.

Internal gravity waves are observed to be easily destabilized. Their eventual breakdown has an important impact on the large-scale circulation of the atmosphere and the oceans by the formation of turbulence and the subsequent momentum, heat and constituent exchanges with the mean flow. To understand and to predict the processes responsible for these instabilities is therefore essential. For the sake of studying the general problem, we analyse an idealized but physically conceivable situation: a single weakly unstable wave which propagates in a dissipative medium and saturates but does not break down. Two layers define the upper atmosphere: the mesosphere and the thermosphere. The lower thermosphere possesses a transition level located at about 100-120 km and called the turbopause where molecular diffusion is comparable to eddy diffusion. Above this level, the former becomes so strong that wave breaking ceases. Unsaturated-wave amplitudes increase with height in the mesosphere. Large-slope waves are likely to break; on the contrary small-slope waves saturate but do not collapse, the saturation zone being confined to a small fraction of the wave (Prusa et al. (1996)). One can therefore consider a small-slope internal wave generated in the troposphere or the middle atmosphere whose amplitude has increased during its propagation through the mesosphere and lower thermosphere, which has not broken and has passed through the turbopause. This wave is then submitted to large-scale instabilities which are saturated by nonlinearity, viscosity and thermal conduction. We assume that during its development due to external forces, the primary wave has grown so slowly that instability only starts when its amplitude has reached the linear-instability threshold. We will see that our study requires a low-frequency and large-amplitude wave in order to select a large-scale instability. Numerous mechanisms can contribute to the instability and saturation of the wave: molecular diffusion, radiative damping, nonlinear wave-wave interactions, and last, the dynamical and convective instabilities which are prevailing. We next review each process briefly and argue that the only possible instability here is dynamical.

Dynamical instability is driven by the wave velocity shear. The most encountered and fastest one is the Kelvin-Helmholtz instability which takes place in a shear layer. Specifically, KH instability attains a maximum growth rate at a wavelength $\lambda \sim 15 h, h$ being the half-depth of the layer. The existence of other similar long-wavelength disturbances destabilizing viscous and stratified shear flows has also been demonstrated (Fritts and Rastogi (1985)). Convection is expected to occur in the mesosphere and lower thermosphere due to unstable lapse rates. One can nevertheless observe overturned waves which saturate but which do not convectively break (Fritts and Rastogi (1985); Weinstock (1990); Lelong and Dunkerton (1998); Sutherland (2001)).

Velocity and temperature profiles of saturated waves show wave-like and long-lasting structures. The turbulence generated in the mesosphere by saturation occupies thin horizontally stratified layers smaller than the vertical wavelength (Fritts et al. (1988a,b); Wu and Widdel (1991)). Upper-atmosphere wind spectra experience different profiles, slopes varying as $m^{-q}$ ( $m$ vertical wavenumber). Anisotropic spectra attributed to gravity wave fluctuations are located for saturated waves at large $m(q=3)$ and for unsaturated ones at small $m(q<3)$ (Weinstock (1990); Tsuda et al. (1991)). Convective breaking is characterized by an isotropic developed turbulence whose spectrum is near $q=5 / 3$. An overturned wave is convectively unstable when the convection time scale is not only smaller than the wave period but also than the other relevant time scales. A wave can convectively break if for instance, it propagates toward a critical layer and its amplitude grows so quickly that dynamical instabilities do not have time to develop. As we will see later on, the convection time is here larger than the viscous-diffusion time. Klaassen and Peltier (1985) highlighted the importance of the Reynolds number on the onset of turbulence in the instability of a KH billow in a stratified shear layer. $R$ varied in their numerical simulations between 300 and 900 with $P r=1$ and $R i=0.07$. The critical Reynolds number has been extrapolated to 220 . Unstable modes derived the major part of their initial growth from shearing conversion. Convection was prevailing at the time of maximum KH wave amplitude (Klaassen and Peltier (1991)).

Lelong and Dunkerton (1998) showed the existence of different instability régimes for a convectively supercritical wave according to its frequency. Shear instability takes precedence over convective instability for near-inertial waves whatever their amplitude and in all disturbance azimuthal directions. The Coriolis force induces a transverse velocity; shear present there cancels convection. For higher frequencies, transverse shear instability dominates with weaker parallel shear instability at larger horizontal scales. Coherent structures appear under the form of KH billows. For frequencies around $3<\omega / f<5$ ( $f$ is the inertial frequency), transverse shear is unable to suppress convection completely and structures evolve toward convection cells. For $\omega / f>5$, transverse convection prevails and the wave collapses. Convective instability is then dominant for high-frequency waves contrary to dynamical instability. 
Convection is characterized by a sudden transition to a tridimensional turbulent flow and the feature consists of counterrotating vortices parallel to the propagation and whose instability vector points in the transverse direction. The third dimension is thus necessary to take convection into account. Nonetheless, a two-dimensional model accurately predicts at what time the onset of breaking occurs for a monochromatic wave or a wave packet (Sutherland (2001)). Andreassen, Wasberg, Fritts and Isler (1994) performed two-dimensional and three-dimensional numerical simulations of gravity wave breaking. They concluded that two-dimensional models can describe wave instabilities and wave-mean flow interactions at large scales. A three-dimensional study is necessary to describe adequately the instability structure accompanying wave breaking, turbulence generation and its effects. Winters \& D'Asaro (1994) carried out nonlinear three-dimensional numerical simulations for the case of a vertically localized and initially two-dimensional gravity wave packet encountering a critical level and compared their results with those obtained in a two-dimensional configuration. They found that the early-time evolution of the critical layer was similar to that in the two-dimensional case, but at late time, transverse convective instabilities developed. Afanasyev and Peltier (2001) analysed a monochromatic wave generated by topography when it propagated in two and three dimensions. Prior to overturning, their three-dimensional results duplicated two-dimensional results to high accuracy because spanwise instabilities showed negligible growth. The growth of these latter onset as the waves began to overturn. The instability could not be characterized as simple gravitational convective instabilities but rather were most probably enhanced by shear with convective instability acting at later stages. Its neglect here on our low-frequency wave whose instability is driven by shear does not turn out to be serious, other instability analyses have been done with such a simplification and yielded satisfying results with respect to experimental studies (Bouruet-Aubertot, Sommeria \& Staquet (1995), Sutherland (2001)).

The nonlinear interactions between a wave and several modes involve primarily resonant interactions when amplitudes are small. The parametric subharmonic instability (PSI) albeit acting at any amplitude cannot prevent the occurrence of shear instability at low horizontal wavenumber or convective instability at large one (Fritts and Rastogi (1985); Klostermeyer (1991)) in a supercritical wave. The small-scale effects of PSI in an inertial gravity wave are analysed in Miyazaki and Adachi (1998). Meek et al. (1985) succeeded in radar-analysing 58 monochromatic waves moving in the mesosphere and irregularly spread over 11 months. Only two of them possessed supercritical amplitudes due to the presence of a critical layer. So, one should not conclude that internal waves do not exist far from their source in the atmosphere. Realistically, monochromatic waves appear relatively rarely. The wind field consists of a superposition of many waves and therefore the waves disperse as they propagate.

Wave packets qualitatively possess the same dynamical properties as monochromatic waves. For instance, if the packet has a weak amplitude, then resonant-interaction driven instabilities are likely to occur. In a numerical study, Yi (1999) showed the importance of the resonant-interaction instability for an internal-wave packet. An upgoing gravity-wave packet was observed to cause two secondary waves to grow from the noise level to a significant intensity. The primary wave packet was deformed as it decayed. So, its amplitude displayed an irregular height structure. Yi also examined the influence of viscosity and concluded that the basic properties of the interactions were the same. The local energy transfer was affected and the repartition of energy density with height was modified. Moreover, an upward propagating wave packet seems to lose with time its horizontal modulation. Prusa (1996) used a two-dimensional numerical model to describe the propagation of an internal-wave packet forced by the troposphere. He observed that the wave field at mesopause altitudes was nearly monochromatic. The dominance of a single horizontal wavelength at high altitudes is a consequence of the dispersion of the wave packet. Like for a monochromatic wave, large-slope wave packets are more sensitive to breaking. The morphology of the wave field in the saturating region is sensitive to the wave slope. The saturation zone increases with the latter. When the slope is order one, saturation involves almost the entire wave. Though nonlinear effects exist, Zhang, Yi and Wang (2000) showed that in their numerical simulations of internal-wave packets propagating in the atmosphere, the time variations of the frequencies of packets were close to those derived from the dispersion relation and the amplitude and phase relations of waveassociated disturbance components were consistent with the predictions of the polarization relation of gravity waves. This indicates that the linear gravity wave theory can be applied extensively in the nonlinear region. In the same way, the linear theory is widely used to describe the dynamics of a monochromatic saturated wave. For example, this theory is able to predict a saturated-wave spectrum compatible with observations (Dewan and Good (1986)). Modulation has also sometimes a stabilizing effect. Campbell and Maslowe (2003) compared the evolutions of a two-dimensional and forced internalwave packet and a monochromatic wave approaching a critical level. They showed that the absorption of 
the disturbance at this level was enhanced for a wave packet and the onset of the nonlinear breakdown was delayed vis-à-vis the monochromatic forcing. The prolonged absorption of the disturbance stabilized the flow to the extent that it was always convectively stable; the local Richardson number remained positive and the regions where $R i<1 / 4$ were reduced. This supports our assumptions.

In this paper, we present a study of a similar stabilization in the context of the negative viscosity theory. This approach for an unstratified and viscous fluid enables one to describe the large-scale instability of a periodic shear flow, the so-called Kolmogorov flow (She (1987), Bondarenko, Gak \& Dolzhansky (1979), Frisch, Legras \& Villone (1996)). When the Reynolds number $R$ is higher than a critical value $R_{0}$, the initially unidirectional flow becomes unstable and a secondary flow appears in the form of a regular system of stationary eddies which remains stable for $R \simeq R_{0}$. The secondary flow obeys a onedimensional amplitude equation whose solution always reaches a stationary state after a very long time for it minimizes a Lyapunov functional. This equation is called the Cahn-Hilliard equation (denoted $\mathrm{CH}$ from now on). Kolmogorov flow instability can be explained phenomenologically by the appearance of a negative eddy-viscosity $\nu_{E}$ when $R>R_{0}=\sqrt{2}$. For a slightly supercritical Reynolds number: $R=R_{0}\left(1+\epsilon^{2}\right)$, with $\epsilon$ a small parameter scaling the wavenumber, $\nu_{E}$ is of order $\epsilon^{2}$ and yields an order $\epsilon^{4}$ growth rate (Frisch et al. (1996)). CH is then derived through an asymptotic expansion with the help of slow space-time variables: $X=\epsilon x$ and $T=\epsilon^{4} t$. The space-periodic solutions of this equation given by pseudo-spectral codes (She (1987); Frisch et al. (1996)) represent nonlinear and soliton-like waves, described by plateaus separated by steep positive and negative gradients: kinks and antikinks which alternate pairwise. Adjacent kinks and antikinks tend to draw nearer, then annihilate, thus lengthening the plateaus and showing an inverse cascade (Legras, Villone, Frisch (1999)).

When Kolmogorov flow is studied for planetary scales in the $\beta$-plane approximation, the large-scale instability is then perturbed by the presence of Rossby waves. Simulations of the modified amplitude equation and analytical theories (Frisch et al. (1996); Manfroi and Young (1999); Legras et al. (1999))

show that the inverse cascade is affected by the dispersive action of the waves, proportional to $\beta \partial_{X^{-1}}$. In the case where $\beta$ is small, the cascade is accelerated, then interrupted at a wavenumber which increases with $\beta$. Numerical simulations of baroclinic small-scale turbulence forcing on large-scale jets taking into account the $\beta$-effect show that these jets become steady in spite of the forcing (Huang and Robinson (1998)). Moreover, the energy transfer toward the large scales is spectrally non-local. This asymptotic method based upon a scale separation and not upon a weak amplitude is assumed to model this nonlocal transfer (Manfroi and Young (1999)). In a stratified and non-rotating medium, Caillol and Zeitlin (2000) demonstrated that under the hypothesis of an infinite number of weakly interacting internal gravity waves, a stationary régime was characterized by a nonlocal transfer of energy in two and three dimensions. In the two-dimensional case, by computing the energy flux, they obtained an inverse cascade. These considerations motivated this work.

The paper is structured as follows. Section (2) displays the basic equations and the different parameters involved in the problem. In section (3), we carry out the linear stability analysis. We show the existence of a true large-scale minimum in the stability diagram provided that on one hand, the ratio stratification over inertia, incidence and friction are weak and on the other hand, the Prandtl number $\mathrm{Pr}$ is close to one. Through a perturbative analysis in the neighbourhood of the threshold, we quantitatively ascertain the effects of each parameter. In the next section (4), we derive the amplitude equation governing the development of a perturbation. In section (5), we describe some numerical results of the evolution of a finite initial perturbation and point out several physical régimes. Then, in section (6), we give an approximate stationary solution of the nonlinear wave and study its stability. Finally, in section (7), we present our conclusions.

\section{Formulation}

We use the Navier-Stokes and entropy equations in the Boussinesq approximation in the plane $(x, z)$, where $z$ denotes the vertical coordinate. The dependent variables are the stream-function $\psi$ and buoyancy $\xi=-g\left(\rho-\rho_{0}\right) / \rho_{0}$ (i.e. variation of the density relative to the mean density $\left.\rho_{0}\right), g$ is the acceleration due to gravity:

$$
\partial_{t} \Delta \psi+J(\Delta \psi, \psi)+\partial_{x} \xi=\nu \Delta^{2} \psi-\chi \Delta \psi+F_{\psi}, \partial_{t} \xi+J(\xi, \psi)-N^{2} \partial_{x} \psi=\kappa \Delta \xi+F_{\xi},
$$

$J(a, b)=\partial_{x} a \partial_{z} b-\partial_{z} a \partial_{x} b$ is the Jacobian. $N^{2}=-g / \rho_{0} d \rho_{0} / d z$ is the square of the constant BruntVäisälä frequency. $\rho_{0}$ exponentially varies with $z$ slowly so that the kinematic viscosity $\nu$, the friction $\chi$ 
and the thermal conduction $\kappa$ are supposed constant in the studied vertical range. The linear wave

$$
\psi=\psi_{0} \cos (k x+m z-\omega t), \quad \xi=-N \psi_{0}\left(k^{2}+m^{2}\right)^{\frac{1}{2}} \cos (k x+m z-\omega t)
$$

is an exact solution of the system provided that a forcing $\left(F_{\psi}, F_{\xi}\right)$ balances the dissipative effects generated by $\nu, \chi$ and $\kappa$. This coherent pattern is taken as the base solution. The wave frequency is $\omega=N k /\left(k^{2}+\right.$ $\left.m^{2}\right)^{1 / 2}, N$ is real and assumed positive for a rightward propagation. The Boussinesq approximation is valid if the vertical wavelength $1 / m$ is less than $2 H, H$ being the density scale height of the atmosphere. Such a wave usually has a small slope $\varpi=k / m$, is quasi-hydrostatic and ubiquituous in the upper atmosphere. In recent years, various ground-based remote sensing facilities have been used to observe the wind velocities, density and temperature of the middle and upper atmosphere. The typical horizontal wavelength varies from tens to hundreds of kilometers. Wave periods vary from tens of minutes to several hours (Meek et al. (1985), Tsuda et al. (1994)). Moreover, this approximation tends to be even more valid at high altitudes. Indeed, Röttger et al. (1990) and Yi et al. (1991) found that in the summer polar mesopause region, the vertical wavelength of gravity waves rapidly decreased with increasing height. This property is as well true for a wave packet arriving at the lower thermosphere (Zhang and Yi (2002)).

The goal of the study is to understand the mechanisms of saturation but is not to focus on the generation. We therefore consider an unbounded propagation zone and the emission zone is discarded. Imposing a forcing at the bottom of a bounded domain, as is often done for modelling the action of the orography, surface waves on the sea or a source located within a layer below the propagation zone, would lead to study a two-dimensional amplitude equation since the wave would be gradually damped as it vertically propagates. We wish to study the effect of damping on the instability but not on the primary wave. The forcing used is therefore the crudest one. Our model is not unrealistic since if one considers for instance, internal waves emitted by unstable shear layers, wave amplitudes may be so large that they can propagate upward several scale heights before dissipating (Fritts, 1984). Wind shear is believed to be one of the most important wave sources. Waves are forced by a Kelvin-Helmholtz type shear instability which can achieve such large amplitudes and kinetic energies that they excite propagating gravity waves in the adjacent stratified layer. Another important internal-wave source in the atmosphere is turbulence, created either by a saturated wave or a turbulent shear flow, for example a boundary-layer turbulence. This turbulence produces momentum and energy fluxes which then appear as forcing terms in the wave motion equations. These fluxes are spatial or temporal averagings of the small-scale and rapid turbulent fluctuations and are therefore coupled. The forcings involving a continuum of wavenumbers are not periodic. We consider the mode with the highest amplitude and exclude all other wavenumbers. Now, if one considers the turbulence generated by saturation, this latter can lead to a momentum-flux convergence and thermal modifications which initiates inertio-gravity waves in a stratified and rotating flow (Walterscheid and Boucher (1984)). More generally, the atmosphere may be highly perturbed by transient external energy sources. The sudden deposition of energy is to generate a disturbance that is geostrophically unbalanced. The adjustment is accomplished by inertio-gravity waves. We can imagine a long-lasting disturbance such that the wave can propagate over several vertical periods before the adjustment is realized.

The preceding system (1) is non-dimensionalised by putting

$$
\psi \rightarrow U m^{-1} \psi, \quad \xi \rightarrow U N \xi, \quad t \rightarrow t /(m U), \quad(x, z) \rightarrow(x / m, z / m)
$$

and thus becomes:

$$
\begin{aligned}
\partial_{t} \Delta \psi+J(\Delta \psi, \psi)+\mathcal{G} \partial_{x} \xi & =\frac{1}{R} \Delta^{2} \psi-\frac{\eta}{R} \Delta \psi-\frac{1+\varpi^{2}}{R}\left(1+\varpi^{2}+\eta\right) \cos \phi \\
\partial_{t} \xi+J(\xi, \psi) & =\mathcal{G} \partial_{x} \psi+\frac{1}{S} \Delta \xi-{\frac{\left(1+\varpi^{2}\right)^{\frac{3}{2}}}{S}} \cos \phi . \quad \text { (b) }
\end{aligned}
$$

$\phi=z+\varpi\left[x-\mathcal{G} t /\left(1+\varpi^{2}\right)^{1 / 2}\right]$ is the wave phase. $U$ is the wave velocity $\left(U=\psi_{0} m\right)$. The following dimensionless numbers appear: the Reynolds number $R=U /(\nu m)$, the Péclet number $S=U /(\kappa m)$ and the inverse Froude number $\mathcal{G}=N /(U m)$. This last number is supposed very small and we will use it to perform our asymptotic analysis. $\operatorname{Pr}=\nu / \kappa$ is the Prandtl number. We introduce $\eta$, a dimensionless parameter, ratio of the viscous time scale to a linear damping: $\eta=\chi /\left(\nu m^{2}\right)$. This unusual term for the internal-wave dynamics models the possible effects of friction due to the presence of boundaries when this problem is undertaken in a tank. In their analysis of the instability of a stratified shear flow, Bachayev and 
Kurganski (1986) measured an experimental critical Reynolds number which was larger than an analytical value obtained after a strong truncation of a periodic solution written in a Floquet basis and related to an unbounded domain. Due to the presence of friction, the saturation of the instability resulted in the appearance of coherent patterns which did not occupy the largest scale. We therefore aim to investigate if the Rayleigh dissipation acts on the critical Reynolds number when the large-scale assumption is used.

\section{$3 \quad$ Linear stability analysis}

This stability analysis departs from the previous studies by the introduction of finite dissipative terms in the equations of motion. The latter had been previously supposed inviscid or evolving at large Reynolds and Péclet numbers. As a result, although we want to focus on large-scale instabilities, we wish to give a general view of the problem in the first subsection by exploring all the wavenumber range for arbitrary stratifications, wave slopes, frictions, Reynolds and Prandtl numbers. We will hence qualitatively describe the evolution of the stability diagram with respect to the variation of these parameters. We will find some previous results again like the importance of the resonant interaction for weak-amplitude waves. The rôle of the Prandtl number whatever the scale will be highlighted. We will bring to the fore the existence of a large-scale instability area for particular values of the parameters. The second subsection will zoom on this zone around the marginal stability, will explain how to choose the right scalings of each parameter yielding a true large-scale minimum and will validate the asymptotic expansion of section (4).

\subsection{The eigenvalue problem and the results}

Let us now examine the stability of a linear wave. We search for a solution of the system (2) as a sum of the wave and a perturbation,

$$
\Psi=\cos \phi+\psi^{\prime}, \quad \Xi=-\left(1+\varpi^{2}\right)^{\frac{1}{2}} \cos \phi+\xi^{\prime} .
$$

Floquet theory on the differential equations possessing periodic coefficients enables us to find a general expression for $\psi^{\prime}$ and $\xi^{\prime}$ as follows:

$$
\left(\begin{array}{c}
\psi^{\prime} \\
\xi^{\prime}
\end{array}\right)=\exp [i(\alpha x+\gamma \phi+\lambda t)] \sum_{n=-\infty}^{\infty}\left(\begin{array}{c}
a_{n} \\
b_{n}
\end{array}\right) \exp (i n \phi),
$$

where $\gamma$ is Floquet's multiplier and $-1 / 2 \leq \gamma \leq 1 / 2$. When $\gamma \neq 0$, the perturbations have a different periodicity to the initial wave. Preceding linear-stability analysis bearing on stratified shear flows showed that same-periodicity disturbances were the most unstable (Khapaev (2000); Young (1999)). We will demonstrate that this result is still valid for an internal wave in the weak-stratification approximation. After injecting this solution in (2), we obtain

$$
\begin{aligned}
\frac{\alpha}{2}\left(b_{n-1}-b_{n+1}\right) & +\frac{\alpha}{2}\left(a_{n-1}-a_{n+1}\right)\left(1+\varpi^{2}\right)^{\frac{1}{2}}+i \mathcal{G}(\alpha+\varpi \bar{n}) a_{n}+\left(\frac{i \mathcal{G} \varpi \bar{n}}{\left(1+\varpi^{2}\right)^{\frac{1}{2}}}-\frac{D_{n}}{S}\right) b_{n}=i \lambda b_{n} \\
\frac{\alpha}{2} & \frac{E_{n-1}}{D_{n}} a_{n-1}+\left(\frac{i \mathcal{G} \varpi \bar{n}}{\left(1+\varpi^{2}\right)^{\frac{1}{2}}}-\frac{D_{n}+\eta}{R}\right) a_{n}+i \mathcal{G}(\alpha+\varpi \bar{n}) \frac{b_{n}}{D_{n}}-\frac{\alpha}{2} \frac{E_{n+1}}{D_{n}} a_{n+1}=i \lambda a_{n}
\end{aligned}
$$

with $D_{n}=\alpha^{2}+\left(1+\varpi^{2}\right) \bar{n}^{2}+2 \varpi \alpha \bar{n}, E_{n}=D_{n}-1-\varpi^{2}$ and $\bar{n}=n+\gamma$.

This eigenvalue problem is tackled by truncating the modes over a range: $-M \leq n \leq M$.

We now present our results. Instability evolves differently in the domains labelled I or II according to $\alpha$ is less or greater than one. Figure 1 (a) shows the effect of $\mathcal{G}$ on the stability of a stationary wave. For increasing stratification, instability appears in I at increasing critical Reynolds numbers $R_{c}>R_{0}$ and wavenumbers $\alpha_{c}$. Instability is confined in a single lobe which then splits up into several pieces. In II, instability appears at infinite $\mathrm{R}$ and $R_{c}$ is a decreasing function of $\mathcal{G}$. The lowest critical Reynolds number is obtained for $\mathcal{G} \simeq 0.5$ and is around $R_{c} \simeq 13.3$, then $R_{c}$ increases with $\mathcal{G}$. Since $R_{c}$ always takes higher values than in I, instability is prevailing in I. For $\mathcal{G}>1$, instability is restricted in I and disappears around a maximum $\mathcal{G}_{c} \simeq 1.2$. We now analyse the effects of the wave slope in Figure 1 (b). For increasing $\varpi, \alpha_{c}$ is strongly shifted toward smaller scales, $R_{c}$ increases and seems to reach a maximum around $\varpi \sim 0.7$. When $\varpi>1$, the instability threshold is no longer located in I, $R_{c}$ decreases and the instability extends very deeply in II. High-frequency waves destabilize at small scales. In spite of the 
presence of a small-scale instability, parallel convection is strongly inhibited by diffusion and instability is driven at large Froude number by shear. Indeed, the maximum convective growth rate is $\left(-g / \rho_{0} \partial \rho / \partial z\right)^{1 / 2}$ whereas the viscous damping is $\nu D_{n} q^{2}$ (equation 5). Their respective dimensionless equivalents satisfy $\left[\left(1+\varpi^{2}\right)^{1 / 2} / \mathcal{G}-1\right]^{1 / 2} \ll D_{n} /(R \mathcal{G})$, which means that convection never occurs. The table (1) summarizes the high destabilizing effect of incidence. We now consider the influence of friction. Its effect is drastic on the presence of a large-scale threshold. $\eta$ rapidly shifts $\alpha_{c}$ toward order 1 wavenumbers in figure 2 (a); as soon as $\eta=0.005, \alpha_{c} \simeq 0.20$ at $R_{c} \simeq 1.6$ and for $\eta=0.05, \alpha_{c} \simeq 0.34$ at $R_{c} \simeq 2.1$. On the contrary, $R_{c}$ slowly varies. Figure 2 (b) shows the competing effects of stratification and friction. The singularity due to stratification is stronger.

We next examine the effects of the Prandtl number. We have compared two marginal curves at $\operatorname{Pr}=1, \mathcal{G}=0.2$ and $\mathcal{G}=0.6$ with the modified curves when respectively $\operatorname{Pr}=1.2$ and $\operatorname{Pr}=5$ in figure 3 (a). In the first case, a narrow negative-growth-rate tongue appears near $\alpha=0$, the curve is cut and the lower-Reynolds part joins $R=0$ at $\alpha=0$. In the second case, the three lobes disappear and form a single and far wider domain containing smaller scales. Instability again begins at $R=0$ for $\alpha=0$. Whatever $R$, long-wavelength perturbations destabilize the wave. Then, we study the stability evolution when thermal diffusion varies from infinity to zero in figures 3 (b) and 4 (a). When $\operatorname{Pr}=0$, the axis $\alpha=0$ belongs to the neutral curve and $R_{c}=0$. A bump appears and narrow tongues emerge at the largest scales with negative growth rates which increase in absolute value. So, paradoxically when $\kappa$ decreases, the instability area shrinks in I. The tongue and the bump draw nearer, then join together when $\operatorname{Pr} \sim 1$. When $\operatorname{Pr}$ goes away from one, the process is inverted; stability is gradually reduced at the largest scales. The curve asymptotes the axis $R=0$ when $\operatorname{Pr}$ approaches infinity; instability tends to occupy all the domain I. The instability area is hence the weakest in I when $\operatorname{Pr}=1$. In II, instability increases with $\operatorname{Pr}$ (cf. figures 4). In numerous papers dealing with numerical studies of internal waves propagating in large-Reynolds-number media, the value $P r=1$ is often encountered because it is a reasonable approximation for gases. We have wished to verify the validity of this assessment at large $R$ in figure 4 (b). Only in the case $\operatorname{Pr}=1$, very large scales are stable. This stability band is nevertheless very narrow. This approximation is thus good at large scale. In II, curves superpose and suddenly separate. An approximate equal distance between curves shows an asymptotic power law evolution of $R$ with $\operatorname{Pr}$.

In summary, we see that internal waves are unstable at any amplitude in spite of viscous and thermal diffusions. The stationary boundary $\mathcal{G}_{c}=1.2$ might be unlikely, the lowest allowed frequency being $f$. Indeed, calculations made for $\varpi=0.01$ showed that up to the resolution limits ( $M=85$ modes), we were able to find instability areas for very high stratification: narrower and narrower lobes for increasing $\mathcal{G}$. They are the locations of resonant interactions between the wave and some modes yielding another internal wave. These interactions are prevailing over other kinds of interactions when amplitudes are weak (Lombard and Riley (1996); Caillol and Zeitlin (2000)) and can even exist for relatively small Reynolds and Péclet numbers. Moreover, instability is strongly dependent on the Prandtl number. $\operatorname{Pr}$ acts whatever the scales. Instability is the weakest at $\operatorname{Pr}=1$ in I. But as soon as $\operatorname{Pr} \neq 1$, an internal wave is always unstable at sufficiently large scale whatever $R$ and $\mathcal{G}$. At last, a large-scale instability threshold is located at low $R$ and is characterized by small $\mathcal{G}, \varpi$ and $\eta$. This latter moves the instability towards the small scales but does not make the critical Reynolds number much higher. Furthermore, $\operatorname{Pr}$ must be close to one in order to have $\alpha_{c} \neq 0$ and $R_{c} \neq 0$. We give in the next subsection the magnitudes of these parameters for an asymptotically small critical wavenumber of the disturbance. We can compare these results with the study of Lombard and Riley (1996). They examined the three-dimensional linear instabilities of a gravity wave propagating with several incidences. They stated like us that the maximum growth rate for $\mathcal{G}<1$ is located at small $\alpha$ but for a zero incidence, they found that the wave is stable when $\mathcal{G}>1$ because instability is convectively driven, which is not the case here. Whatever incidence, they observed that for high $\mathcal{G}$, the instability is explained by three-mode resonant interactions. But, for weaker $\mathcal{G}$, the interaction concerns more modes which do not belong anymore to the initial wave plane. These modes become thus three-dimensional and possess the highest growth rates. Internal-wave instability is as a result three-dimensional for small $\mathcal{G}$ and large $R$ (Klostermeyer (1991)). In our study, three-dimensional convective instabilities do not arise due to the low Reynolds number.

\subsection{Study of the critical zone of stability}

We wish, in this subsection, to focus on the large-scale marginal stability. We successively examine the effects of $\mathcal{G}, \operatorname{Pr}, \eta, \varpi$ and $\gamma$, then give their correct scalings as functions of $\alpha$. We solve this eigenvalue problem analytically by truncating at $M=1$. The hypothesis of strong truncation is valid 
owing to a high diffusion in this zone. The search of the eigenvalues comes down to determining the real roots of a $\lambda$-polynomial whose complex coefficients depend on the above parameters. The relationship obtained between $R, S, \alpha \ldots$ being too complex, we use the weak-stratification assumption to obtain $R$ perturbatively. $\lambda$ and $R$ are then expanded following $\mathcal{G}$ :

$$
\lambda=\mathcal{G} \lambda^{(1)}+\mathcal{G}^{3} \lambda^{(3)}+\ldots, \quad R=R^{(0)}+\mathcal{G}^{2} R^{(2)}+\ldots
$$

For more details, the complete study can be found in (Caillol (2000)).

\subsection{Effects of stratification $\mathcal{G}$ and Prandtl number $\mathrm{Pr}$}

As equations (2) are uncoupled at the leading order, $R^{(0)}$ does not depend on $\operatorname{Pr}$ and is identical to the marginal Reynolds number of an unstratified shear flow (Batchayev and Kurganskiy (1986))

$$
R^{(0)}=\sqrt{2} \frac{1+\alpha^{2}}{\left(1-\alpha^{2}\right)^{\frac{1}{2}}} .
$$

Instability is confined to the domain I and $R^{(0)}>R_{0}$. The study of the behaviour of $R^{(0)}+\mathcal{G}^{2} R^{(2)}$ with $\alpha$ shows the existence of a minimum if and only if $\operatorname{Pr}$ is close to one. The marginal perturbation is a linear wave of frequency $\lambda^{(1)}=-\alpha$ when $\operatorname{Pr}=1$, otherwise $\lambda^{(1)}$ diverges for $\alpha$ small, of order $\epsilon$. Consequently, we wish to get a disturbance which is at the first order a wave. The condition is realized provided $\operatorname{Pr}$ is taken: $\operatorname{Pr}=1+\epsilon^{2} \delta P r . \lambda^{(1)}$ and $R^{(2)}$ are then expanded following $\epsilon$ :

$$
\begin{aligned}
\lambda^{(1)} & =-\alpha-\frac{\delta P r}{2 \alpha} \epsilon^{2}+O\left(\epsilon^{2}\right), \\
R^{(2)} & =\frac{1}{\sqrt{2} \alpha^{4}}+\frac{5}{2 \sqrt{2} \alpha^{2}}-\frac{\delta P r \epsilon^{2}}{\sqrt{2} \alpha^{4}}-\frac{\delta P r^{2} \epsilon^{4}}{4 \sqrt{2} \alpha^{6}}+O(1) .
\end{aligned}
$$

After (8), $\mathcal{G}$ must be of order $\epsilon^{3}$ so that $R$ may possess a minimum; the critical wavenumber and Reynolds number are hence

$$
\begin{aligned}
\alpha_{c} & =\left(\frac{2}{3}\right)^{\frac{1}{6}} \mathcal{G}^{\frac{1}{3}}+O\left(\frac{\delta r^{2} \epsilon^{4}}{\mathcal{G}^{\frac{1}{3}}}, \mathcal{G}, \delta \operatorname{Pr}^{\frac{1}{3}} \epsilon^{2}\right), \\
R_{c} & =\sqrt{2}\left(1+\left(\frac{3}{2}\right)^{\frac{5}{3}} \mathcal{G}^{\frac{2}{3}}\right)+O\left(\delta \operatorname{Pr}^{\frac{2}{3}} \epsilon^{2}, \mathcal{G}^{\frac{4}{3}}, \delta \operatorname{Pr}^{2} \epsilon^{4}\right) .
\end{aligned}
$$

$\operatorname{Pr}$ has thus a very small effect (of order $\epsilon^{4}$ ) on $R_{c}$. We notice a divergence of $R^{(2)}$ for $\alpha$ tending to 0 . $R^{(2)}$ is of same order as $R^{(0)}$ when $\mathcal{G}^{2} / \alpha^{4}=O(1)$, so by rescaling the wavenumber with $K=\alpha / \mathcal{G}^{1 / 2}$, we can better capture the behaviour of the neutral curve for $\alpha \leq \alpha_{c}$. The related inner expansion is given in Appendix A. The singularity is now present at the first order. This scaling is valid when stratification prevails over the other parameters on the singularity in 0 . The scalings of $\eta, \operatorname{Pr}$ and $\gamma$ are different when $K=O(1)$ from those chosen to locate the threshold at a $O(\epsilon)$ critical wavenumber. The condition on $\operatorname{Pr}: \operatorname{Pr}-1=O(\mathcal{G})$ is thus very drastic if one wishes to get a smooth and decreasing function $R(\alpha)$ for $\alpha \rightarrow 0$ and $R_{c} \neq 0$.

\subsubsection{Effects of incidence $\varpi$, friction $\eta$ and disturbance periodicity $\gamma$}

Slope increases mode coupling and prevents a rapid convergence of the series (4), so the outer expansion fails to model the marginal stability for $\varpi=O(1)$. The inner expansion is valid for $\varpi<7^{-1 / 2}$ (cf. Appendix A). For $\varpi=O\left(\epsilon^{2}\right)$, as will be the case later on, the effects of $\varpi$ on $\lambda^{(1)}, \alpha_{c}$ and $R_{c}$ are negligible. A $O(\epsilon)$ critical wavenumber leads to conditions on $\alpha_{c}, \eta$ and $\gamma$ :

$$
-2 \mathcal{G}^{2}+\alpha_{c}^{2}\left(3 \alpha_{c}^{4}-9 \gamma^{2}-\eta\right)=0 .
$$

The eigenmode $\lambda^{(1)}=-\alpha-\eta / \alpha+O\left(\epsilon^{5}\right)$ does not depend on $\gamma$. Friction weakly alters $\lambda^{(1)}$ and $R_{c}$ but strongly $\alpha_{c}$ (cf. figures 2) as the numerical analysis has shown. In figure 5 (a), we have plotted different profiles of marginal stability by using the above asymptotic expressions of $R(\alpha)$. We observe a good agreement in the prediction of the critical zone by the outer expansion. However, due to the strong truncation, this cannot predict the neutral curve for $\alpha>\alpha_{c}$. The inner expansion can fit very well the 
numerical curve for $\alpha<\alpha_{c}$ and this for any $R$. When $\eta \neq 0$, a discrepancy exists due to the competing effects of stratification and friction; the inner expansion is only correct when $\mathcal{G}$ prevails over $\eta$.

The periodicity of the disturbance unexpectedly strongly inhibits the appearance of a large-scale instability. $\alpha_{c}$ and $R_{c}$ increase with $\gamma$ more slowly than with $\mathcal{G}$ or $\eta$ but for $\gamma$ varying up to $1 / 2, \alpha_{c}$ reaches order-one values (cf. figure $5(\mathrm{~b})$ ). In this figure, we have plotted the minimum Reynolds numbers firstly obtained in (3.1) and secondly by a secant method solving the equation $d R / d \alpha=0$ with $R=R^{(0)}+\mathcal{G}^{2} R^{(2)}$ for $\gamma$ varying between 0 and $1 / 2$ and different values of $\mathcal{G}$. $\left(\alpha_{c}, R_{c}\right)$ very weakly depends on $\mathcal{G}$. For $\gamma$ reaching a value close to 0.35 , a bifurcation occurs. All points $\left(\alpha_{c}, R_{c}\right)$ cluster around $(0.41-0.42,6.6-6.8)$. This bifurcation cannot be described by our truncated model. Such a bifurcation was observed for an unstratified shear flow around the same value of $\gamma$ (Young (1999)).

\subsubsection{Summary and relevance}

This perturbative approach has demonstrated several observations made in the previous subsection. It defines a large-scale instability threshold located at $R_{c} \geq R_{0}$ provided that $\mathcal{G}, \eta$, $\varpi$ and $\gamma$ are weak. For small wavenumbers, it is then possible to find analytical expressions of the marginal Reynolds number as a function of $\alpha$ and the different parameters. The latter thus act in an asymptotically small way and the internal-wave marginal stability is close to that of an unstratified shear flow. It has been also shown that $\operatorname{Pr}$ must be very close to one in order to have a true minimum $R_{c}$ different from zero.

Furthermore, this analysis can only be applied to fluids like air but not sea water where $\operatorname{Pr} \sim 8$. Close values of Reynolds and Péclet numbers are not restrictive for thermal conduction damps internal waves approximately by an equal amount as molecular viscosity in the upper atmosphere (Kato (1980)). Besides, a dispersive effect has long been attributed to both these dissipations (Lamb (1945)). On the contrary, the condition on $\gamma$ is more stringent. Indeed, this study has revealed that $\alpha_{c}$ strongly depended on $\gamma$, which had not been reported previously. If for instance, we discuss possible applications of these results to the upper atmosphere, $\nu$ and $\kappa$ reach large values there because density is very small. Our model implies two constraints: a low-frequency wave and a large-Froude-number wave. This latter corresponds in our study to a small-vertical-scale and large-amplitude wave propagating in a moderately stratified flow. Such waves cannot persist above a certain height because they are damped (see in particular the graph 6 in Hines (1963)). In his study of an upward going internal-wave packet, Prusa (1996) calculated an estimate of the altitude at which wave breaking ceases, that is where external dissipation becomes faster than the dissipation rate derived from saturation arguments. The corresponding dissipation rate to saturation of the wave is $\tau_{b}^{-1}=c_{g z} /(2 H)$ with $c_{g z}$ the vertical group velocity. This prediction agreed very well with his numerical model. In our case, if we compare the viscous dissipation rate for a quasihydrostatic wave: $\nu m^{2}$ and $\tau_{b}^{-1}=N \alpha /\left(2 m^{2} H\right)$, the condition of non-breaking yields: $2 H m>R \mathcal{G} \varpi$ $\left(\simeq \epsilon^{5}\right.$ in our study). This inequality is consistent with the Boussinesq approximation. The former is valid above the turbopause and, as a consequence, waves no longer break.

\section{Supercritical motion}

We now search for solutions of (2) in the form of a perturbative expansion. We find the same scalings as in subsection 3.2. The evolution equations related to the leading-order buoyancy and vertical velocity are displayed. Both these equations combine in a single amplitude equation which will be studied numerically and analytically in the following sections. We next mention some energetical features of this particular motion and point out the effects of a rotating medium on the dynamics.

\subsection{Long-wave expansion}

Reynolds and Péclet numbers take supercritical values: $R=R_{0}\left(1+\epsilon^{2}\right), S=S_{0}\left(1+\epsilon^{2}\right)$. The solution (3) is expanded following $\epsilon$ : $\psi^{\prime}=\psi^{(0)}+\epsilon \psi^{(1)}+\ldots$ The details of the calculations are relegated to Appendix B. Equation (B2) induces a first relation obtained at the order $\epsilon^{4}$ between $\left\langle\xi^{(2)}\right\rangle$ and $\psi^{(0)}(\langle\rangle$ denotes an average over $\phi)$ :

$$
R_{0} \partial_{X}^{2}\left\langle\xi^{(2)}\right\rangle+G \partial_{X} \psi^{(0)}-R_{0} \partial_{X}^{4} \psi^{(0)}+\frac{1}{2} R_{0} \delta \operatorname{Pr} \partial_{X}^{2} \psi^{(0)}=0
$$


Equation (B1) yields the second relation derived at the order $\epsilon^{6}$ :

$$
\begin{gathered}
\partial_{T X} \psi^{(0)}+\frac{3}{2} R_{0} \partial_{X}^{5} \psi^{(0)}-R_{0}^{3} \partial_{X}\left[\partial_{X}^{2} \psi^{(0)}\left(\partial_{X} \psi^{(0)}\right)^{2}\right]= \\
-G\left\langle\xi^{(2)}\right\rangle-R_{0} \partial_{X}^{3} \psi^{(0)}-r \partial_{X} \psi^{(0)}+\frac{1}{2} \mu R_{0}^{2} \partial_{X}\left(\partial_{X} \psi^{(0)}\right)^{2}
\end{gathered}
$$

Stratification, friction, incidence and Prandtl number scale as follows

$$
\mathcal{G}=\epsilon^{3} G, \quad \eta=\epsilon^{4} r R_{0}, \quad \varpi=\epsilon^{2} \mu, \quad \operatorname{Pr}=1+\epsilon^{2} \not r .
$$

$\mathcal{G}$ has the same scaling as the case of the only velocity field basic flow (Balmforth and Young (2002)). The critical Reynolds number is still $R_{0}=\sqrt{2}$. The instability starting from $R>R_{c}$, conditions appear on $G$ and $r$ : when $R_{c}$ is firstly given by stratification, (10) implies $G<G_{c}=(2 / 3)^{5 / 2} \simeq 0.363$; when $R_{c}$ is given by friction, (11) leads to $r<r_{c}=\left(2^{1 / 2} 3\right)^{-1} \simeq 0.2357$. From $\psi$ and $\xi$ expressed at the lowest orders, we can easily show that the flow is decomposed between a z-dependent amplitude- and phase-modulated wave and the $X$-evolving nonlinear wave.

We now outline some energetical aspects related to this motion. Averaging over the horizontal direction when periodic boundary conditions are assumed, the buoyancy flux is

$$
\langle\overline{\Xi w}\rangle=-\frac{\mathcal{G}}{R_{0}} \overline{\langle\psi\rangle^{2}}+O\left(\epsilon^{4}\right) .
$$

The overbar denotes the horizontal average over a period. A negative flux implies a loss of the disturbance kinetic energy in addition to the loss due to diffusion. The balance is given by a positive shear energy production. The mechanical equilibrium of the atmosphere has enhanced, indeed mean potential energy $\left\langle\Xi^{2}\right\rangle$ has decreased in spite of a negative buoyancy flux (its variation is $\left\langle\Xi^{2}\right\rangle=-\langle w\rangle^{2}+O\left(\epsilon^{4}\right)$ ); the balance is provided by thermal dissipation and by a weakly positive or negative density-gradient instability production. The shear driven instability is thus highlighted (Lombard and Riley (1996); Lelong and Dunkerton (1998)).

Let us examine the vertical energy flux carried by the wave. The total mean rate of working by the fluid below any level on the fluid above is $\langle\overline{P w}\rangle$, where $P$ is the pressure. When a hypothetical stationary state is reached, the energy flux is

$$
\langle\overline{P w}\rangle=-\frac{\mathcal{G}}{\varpi}\left[\overline{\langle w\rangle^{2}}+\frac{1}{2}(P r-1) \overline{\langle\psi\rangle^{2}}\right]+O\left(\epsilon^{4}\right) .
$$

The details of the derivation are in Appendix C. When $\delta P r \geq 0$, the flux direction is given by the vertical group velocity: $-\mathcal{G} \varpi /\left(1+\varpi^{2}\right)^{3 / 2}$. However, when $\delta \operatorname{Pr}<0$ (case of air), an upward propagating wave can create an upward flux. When $\varpi \rightarrow 0$, the terms inside the brackets vanish, if $\delta \operatorname{Pr}>0$, a true stationary state can be only reached if we assume the presence of a coherent pattern moving with a celerity $G+c$. $c$ is then expressed at the leading order by

$$
c=\frac{1}{2} G \delta \operatorname{Pr} \frac{\overline{\left.\psi^{(0)}\right)^{2}}}{\overline{\left(\partial_{X} \psi^{(0)}\right)^{2}}} .
$$

In the general case of a constant-speed motion, the vertical-flux direction can no longer be predicted.

\subsection{The amplitude equation}

Combining (12) and (13), our problem can reduce to the study of a single equation that is rewritten, using the vertical velocity $w=-\partial_{x} \psi^{(0)}$ and reemploying $x$ and $t$ to describe slow space and time :

$$
\begin{aligned}
\partial_{t} w= & \partial_{x}\left[\left(\lambda_{1} w^{2}-\lambda_{2}\right) \partial_{x} w\right]-\lambda_{3} \partial_{x}^{4} w \\
& -\mu \partial_{x} w^{2}+\frac{G^{2}}{R_{0}} \partial_{x}^{-2} w-r w-G \partial_{x} w+\frac{1}{2} G \delta \operatorname{Pr}_{x}^{-1} w,
\end{aligned}
$$

with $\lambda_{1}=2 \sqrt{2}, \lambda_{2}=\sqrt{2}$ and $\lambda_{3}=3 / \sqrt{2}$.

The first line is $\mathrm{CH}$. The second possesses a quadratic nonlinearity due to the slope and linear damping terms generated by stratification and friction. We find again the two terms determined in the linear 
stability analysis (cf. equation (7)), the advection $-G \partial_{x} w$ (removed by changing referentials $x \rightarrow x-G t$ ) and the dispersion acting as $\partial_{x}^{-1} w$, in the same way as the Rossby wave dispersive effect. We can thus expect the $\mathrm{CH}$ eddy-viscosity driven inverse cascade to be arrested by both the dissipative actions of stratification and friction, and by dispersion. The presence of a quadratic term guarantees the existence of chaotically behaving solutions (Sivashinsky (1985)) for sufficiently small $r$ and $G$. Dispersion and slope prevent the equation from being reformulated with a Lyapunov functional (Balmforth and Young (2002)). As a result, when $\delta P r=0$ and $\mu=0$, the solution tends for long times to a steady state.

\subsection{Rotation}

This study concerns a near-inertial wave. So, the effect of the Coriolis force should be taken into account. One can measure its effects on a fluid according to the Rossby number given by $R o=U m / f$. In this approach, we can express $R o$ via $\mathcal{G}$ and $\tau=f / N: R o=(\tau \mathcal{G})^{-1}$. As a result, $R o \simeq \epsilon^{-3} / \tau$ and $\tau \simeq 10^{-2}$ at mean latitude in the upper atmosphere, so $R o$ takes very large values due the large-Froude-number condition and rotation influence is weak.

If we conserve the two-dimensionality assumption and the same approach, technically to introduce rotation comes down to couple the dimensionless transverse-velocity equation to the equations (2)

$$
\partial_{t} v+J(v, \psi)=-\frac{1}{R o} \psi_{z}+\frac{1}{R} \Delta v-\frac{\eta}{R} v+\frac{\tau}{R}\left(\frac{1+\varpi^{2}}{\tau^{2}+\varpi^{2}}\right)^{\frac{1}{2}}\left(1+\eta+\varpi^{2}\right) \cos \phi
$$

and to add $R o^{-1} v_{z}$ at the r.h.s of (2) (a). The primary wave is modified of the following way

$$
\zeta=-\varpi\left(\frac{1+\varpi^{2}}{\tau^{2}+\varpi^{2}}\right)^{\frac{1}{2}} \cos \phi, \quad v=\tau\left(\frac{1+\varpi^{2}}{\tau^{2}+\varpi^{2}}\right)^{\frac{1}{2}} \cos \phi, \quad \omega=\mathcal{G}\left(\frac{\tau^{2}+\varpi^{2}}{1+\varpi^{2}}\right)^{\frac{1}{2}} .
$$

When $\varpi$ is close to zero, the basic density vanishes, the initial pattern is simply a $z$-periodic velocity field oscillating with a near-inertial frequency. Rotation affects the amplitude equation according to $\tau$. If $\tau=o\left(\epsilon^{2}\right)$, rotation does not alter it. If $\tau=O\left(\epsilon^{n}\right), n=0,2$, the different parameters scale like: $T=\epsilon^{2+n} t$, $\varpi=O\left(\epsilon^{n}\right), \operatorname{Pr}-1=O\left(\epsilon^{n}\right), \eta=O\left(\epsilon^{2+n}\right)$ and $R=R_{0}\left(1+\epsilon^{n}\right)$. If $\tau=\epsilon^{2} \tau_{0}$, then the constant advection is multiplied by $\mu\left(\tau_{0}^{2}+\mu^{2}\right)^{-1 / 2}$ in the same way as the $\delta P r$-dispersive term and consequently, the wave speed (17). The effect of rotation is to decrease dispersion and wave propagation. These latter vanish if $\mu=0$, which is more realistic since the basic flow is not a dispersive wave any more. For a more rapid rotation, when $n=1$, in a non-atmospheric context, the evolution equation is (18) without $\lambda_{1}$, $\lambda_{3}$ and $G^{2}$-damping terms. Negative-viscosity and slope driven instabilities can be only saturated by friction and dispersion. A coherent structure is therefore more unlikely to appear. Three-dimensionality would certainly enrich dynamics owing to the advection by the transverse velocity. Many studies of long nonlinear waves in stratified and rotating flows have been undertaken particularly in the ocean and many of the theoretical results can be applied to atmospheric motions (Grimshaw et al. (1998)). The main assumption, a long wavelength with respect to the depth of the shear layer is valid in our case. Rotation is for instance, modelled in the Ostrovsky equation which reduces to a Korteweg-de Vries equation (KdV, quadratic nonlinearity) or a modified Korteweg-de-Vries equation (MKdV, cubic nonlinearity) when it is discarded. Rotation introduces a new dispersive term which makes the former equation non-integrable. Periodic steady solutions nevertheless exist and it is possible to describe them analytically when rotation is weak.

\section{Numerical simulations of the amplitude equation}

We give here space-periodic numerical solutions of the equation (18). After the previous section, we expect a steady state to be attained at large scales. We show the possibility of obtaining periodic steady states corresponding to long nonlinear waves for small $G$ and $\mu$. This section makes the assumptions used in the analytical study of the following section valid. It also reveals the presence of chaos in the simulations for appropriate values of the slope vis-à-vis the inverse Froude number.

The spatial integration is performed by a pseudo-spectral code. The temporal integration is done using an Adams-Bashforth scheme. The spatial period $L$ is: $L=2 \pi p, p$ integer. Fourier modes are truncated between $-M \leq \alpha \leq M,(M=128$ usually $)$. The initial conditions for each run are a random noise. $\lambda_{3}$ and $G^{2}$-dissipations are treated by an exponential damping. The use of the Adams-Bashforth scheme introduces slow instabilities due to an oscillating motion with the frequency $\omega=\operatorname{poPr} G /(2 \alpha)$. 
In order to avoid them, the time step $\delta T$ must satisfy the condition $8 T^{3}(G \delta P r)^{4} p^{6} \ll 16$ (Frisch et al. (1996)). As $G$ is taken small, this condition is always assured. The most stringent condition occurs when the incidence is present: $8 T<p /\left(2 \mu \max \left|\partial_{x} v\right|\right)$.

We now give the results of our simulations. We have represented the temporal evolution of the vertical velocity and the buoyancy of an initial perturbation over a horizontal period and the corresponding evolution of the disturbance energy distribution: $1 / 2\left(\partial_{X} \psi^{(0)}\right)^{2}$ in the smallest wavenumbers. Figures 6 (a) \& (b) show the CH unstable kink-antikink structure (She (1987) and Frisch et al. (1996)). The energy is mainly concentrated on a single mode which changes suddenly for a smaller one. We examine the effects of each parameter on the evolution of this cascade. Figures $6(\mathrm{c}-\mathrm{d})$ illustrate the braking of the cascade by $G$, at $t=10000$, the cascade is stopped at $\alpha=3$ while energy has saturated as soon as $t \simeq 2000$. Friction has a similar effect. For a deeper study of friction on the Kolmogorov flow, the reader is invited to refer to Legras and Villone (2003). Comparing the terms in $\partial_{x}^{4}, G^{2} \partial_{x}^{-2}$ and $G \delta \operatorname{Pr} \partial_{x}^{-1}$, we deduce that stratification dissipates with a negligible strength when $G \leq p^{-3}$ and dispersion is prevailing on stratification for $\alpha>R_{0} G p / \delta P r$. In figures 7(a-b), the dispersive effect is added for such a weak level of stratification: for $\delta P r=1$, the cascade stabilizes at the mode $\alpha=2$, which appears later than in 6 (b). When dispersion is higher, $\delta P r=10$ in figure 8 (a), the last mode $\alpha=3$ appears earlier. The acceleration of the cascade by dispersion has been pointed out for the $\beta$-effect modified CH (Frisch et al. (1996)). This effect is here weaker due to the competing actions of stratification and dispersion. Buoyancy noticeably increases during the cascade due to dispersion (cf. equation 12); meanwhile velocity amplitude quickly reaches a quasi-stationary state. The patterns move with a propagation speed in the same way as the primary wave if $\operatorname{Pr}>1$ but can be inverted if $\operatorname{Pr}<1$ and if the cascade attains very large scales (cf. equations 7,17$)$. We will give an expression of the nonlinear-wave speed in the following section. The halt of the cascade is less efficient when $\operatorname{Pr}<1$; the same run obtained for $\delta P r=-10$ shows that the cascade is arrested later than in figure 8 (a) reaching the mode $n=2$. However, the acceleration is more efficient, the mode 3 appears earlier at $t=500$. The counter-propagation with respect to the primary wave of near-internal waves superimposed to the kinks increases the kink-antikink coupling.

Figures $7(\mathrm{c}-\mathrm{d})$ and 8 (b-c) show the strong effect of $\mu$ on the cascade. Mode transitions and stabilization occur much earlier in figure 7 (d) where $\mu=0.01$ than in 7 (b). The process is enhanced in figure 8 (b) where $\mu=0.1$. When $\mu=0.5$ in figure 8 (c), the cascade is stopped at $\alpha=3$ and the energy is strongly damped. In figures 9 , we can better observe the effects of $\mu$ on our coherent structures. The flow is a succession of rolls of counterrotating vortices. When $\mu=O(1)$ in figure 8 (d), structures lose their coherences, it is nevertheless possible to reach a stationary state for $\mu=1$ (cf. figure 9 (c)) but not for $\mu=2$. A chaotic régime thus appears for particular values of $G$ and $\mu$. Increasing $G$ delays and stops the emergence of chaos. The threshold value of $G$ is difficult to determine due to the necessity of long-time runs. For $\mu=4$ in figures 9 (d) and 10, a new structure however emerges of larger wavenumber, smaller amplitude and which does not possess a plateau. This pattern is likely to be a manifold attained at very long times for flows with $\mu=O(1)$ but which appears earlier and earlier for larger incidences. The order $\epsilon^{2}$ energy is very weak (cf. figure 10 (b)). Slope has a damaging effect on the convergence of this expansion. As a result, two régimes can be distinguished for high $\mu$ : when $\varpi=\epsilon^{2} \mu \ll 1$, the linear wave is quasi-conserved, the disturbance remains weak (cf. figure 9 (d)). In the contrary case, the disturbance strongly grows. This large-scale approach cannot model the small-scale turbulence which should emerge to damp out the instability in the standard saturation process and the flow blows up.

This section has showed that the wave instability leads to the formation of coarsening patterns. However, this process easily stops before the largest spatial scale of the domain is reached for weak values of $G, \mu$ and $r$ and yields steady states. In the following section, we analytically study such a state.

\section{A particular stationary nonlinear wave}

The relaxation to steady states of the wave instability for small parameters enables us to determine a perturbative stationary solution of (18) with respect to a stationary $\mathrm{CH}$ solution. Then, we undertake the study of the stability of such a solution which yields the critical values of the inverse Froude number above which this steady state stabilizes. These values are very weak as the numerical results of the previous section have shown. We demonstrate that $\operatorname{Pr}$ stabilizes more efficiently the nonlinear wave than $G$. We use Kawasaki and Ohta theory (Kawasaki and Ohta (1982)) and the more specific Legras theory which has been applied to the effect of Rossby waves on the CH inverse cascade (Legras et al. (1999)). The detailed study related to this section can be found in (Caillol (2000)). The dispersive effect encountered 


\subsection{The approximate solution}

We use an approximate solution which remains valid when the distance between the kinks is sufficient, the error being inversely proportional to the exponential of this separation. We focus on solutions which are spatially $L$-periodic. The coherent pattern is constituted by $n$ pairs of alternated kinks and antikinks separated by an equal distance $\Lambda=L / n$. Let us consider a base solution $\bar{w}^{(0)}$ satisfying the stationary $\mathrm{CH}:-\partial_{x}^{2} \bar{w}^{(0)}+U^{\prime}\left(\bar{w}^{(0)}\right)=0$ with $U(w)=s^{2} /\left(2 \Gamma^{2}\right) w^{4}-s^{2} w^{2}$. The constants have the values : $s=3^{-1 / 2}$ and $\Gamma=(3 / 2)^{1 / 2} \cdot \bar{w}^{(0)}$ is built in the spatial range $[-\Lambda / 4,3 \Lambda / 4]$ of the following way

$$
\begin{array}{ll}
\bar{w}^{(0)}=\Gamma \tanh s x \quad \text { while } & -\frac{\Lambda}{4}<x<\frac{\Lambda}{4}, \\
\bar{w}^{(0)}=-\Gamma \tanh s\left(x-\frac{\Lambda}{2}\right) \quad \text { while } \quad \frac{\Lambda}{4}<x<\frac{3 \Lambda}{4} .
\end{array}
$$

If the slope is not assumed small, asymptotic $\mathrm{CH}$ solutions modified by the quadratic term can be determined:

$$
w= \pm \Gamma \sqrt{1 \mp \mu \Gamma^{2}} \tanh \left[s \sqrt{1 \mp \mu \Gamma^{2}} x\right] .
$$

These solutions locally correspond to a kink and an antikink which cannot be matched together, which explains the existing slope of the plateaus when an incidence is applied. The numerical computation showed us that a kink-antikink structure was inhibited from order-one $\mu$ (cf. figure $8(\mathrm{~d})$ ). The maximum incidence for obtaining our nonlinear waves is thus approximately $\left|\mu_{c}\right|=\Gamma^{-2}=2 / 3$.

\subsection{The perturbative order-one solution}

The perturbative solution $\bar{w}(x-c t)$ like other physical quantities is expanded following the amplitudes of $G, \mu$ and $r$ (of order $\delta$ ): $\bar{w}=\bar{w}^{(0)}+\bar{w}^{(1)}+\bar{w}^{(2)}+O\left(\delta^{3}\right) . \bar{w}^{(1)}$ satisfies the following equation :

$$
\lambda_{3} \mathcal{F}\left[\bar{w}^{(1)}\right]=\frac{1}{2} G \delta \operatorname{Pr} \partial_{x}^{-3} \bar{w}^{(0)}+c_{1} \partial_{x}^{-1} \bar{w}^{(0)}-r \partial_{x}^{-2} \bar{w}^{(0)}-\mu \partial_{x}^{-1}\left(\bar{w}^{(0)^{2}}-{\overline{w^{(0)}}}^{2}\right) .
$$

$\mathcal{F}$ is defined by $\mathcal{F}(g)=\partial_{x}^{2} g-U^{\prime \prime}\left(\bar{w}^{(0)}\right) g \simeq-4 s^{2} g$ for the distance between 2 kinks is large.

The nonlinear-wave speed is determined after (17):

$$
c_{1}=\frac{\delta P r G}{2} \frac{\frac{\Lambda^{2}}{48}-\frac{\pi^{2}}{12 s^{2}}+\frac{A}{\Lambda s^{3}}}{1-\frac{4}{\Lambda s}}, \quad A=2.4041 \ldots
$$

By using equation $(\mathrm{C} 1)$, we can evaluate the mean vertical flux of energy. At leading order in $\delta,\langle\overline{P w}\rangle$ is of order $\delta^{2} \epsilon^{3}$ and involves $\bar{w}^{(2)}$ which is calculated with the same approximations. Keeping only the larger polynomial term in $\Lambda$, we obtain

$$
\langle\overline{P w}\rangle=\epsilon^{3} \frac{9}{5 s} \delta \operatorname{Pr} c_{g z}\left(\frac{\Lambda}{16}\right)^{3}+O\left(\epsilon^{3} \mu G \delta \operatorname{Pr} \Lambda^{2}\right),
$$

where the vertical group velocity is $c_{g z}=-\mu G$. The energy flux has a higher amplitude than the unsaturated-wave flux of order $\epsilon^{5}$. When $\operatorname{Pr}<1$, it is in the opposite way than this latter.

\subsection{Stability of the solution}

We here consider a perturbation $\delta w$ to the stationary solution: $w=\bar{w}+\delta w$. We neglect the slope $\mu$. The variable $\varphi=\partial_{x}^{-1} \delta w$ is governed by the equation

$$
\partial_{t} \varphi=\mathcal{L} \varphi
$$

with $\mathcal{L}=-\lambda_{3} \partial_{x}\left[\partial_{x}^{2}-U^{\prime \prime}(\bar{w})\right] \partial_{x}-r+\delta \operatorname{Pr} G / 2 \partial_{x}^{-1}+c \partial_{x}+G^{2} R_{0}^{-1} \partial_{x}^{-2}$.

We expand $\mathcal{L}$ and the eigenvalues $\sigma$ :

$$
\mathcal{L}=\mathcal{L}_{0}+\mathcal{L}_{1}+\mathcal{L}_{2}+O\left(\delta^{3}\right), \quad \sigma=\sigma_{0}+\sigma_{1}+i \vartheta_{1}+\sigma_{2}+i \vartheta_{2}+O\left(\delta^{3}\right)
$$


$\sigma$ contains imaginary terms due to the presence of dispersion. We employ the orthogonal eigenfunctions $\varphi_{a}$ and $\varphi_{b}$ of $\sigma$ with respect to the operator $\mathcal{L}$. They are also $\delta$-expanded and satisfy

$$
\begin{aligned}
\mathcal{L}_{0} \varphi_{a} & =\sigma_{0} \varphi_{a} \\
\mathcal{L}_{0} \varphi_{a 1}+\mathcal{L}_{1} \varphi_{a} & =\sigma_{0} \varphi_{a 1}+\sigma_{1} \varphi_{a}-\vartheta_{1} \varphi_{b}, \ldots
\end{aligned}
$$

and similar equations for $\varphi_{b i} . \sigma_{0}$ is given by (Legras et al. (1999))

$$
\sigma_{0}=\frac{128 s^{3} \lambda_{3} e^{-s \Lambda}}{\Lambda} \sin ^{2} \frac{\pi m}{n} \frac{1}{1-\frac{2\left(\cos \theta_{m}+1\right)}{\Lambda s}}, \quad \theta_{m}=\frac{\pi m}{n} .
$$

$\Lambda$ being large, $\sigma_{0}$ is positive whatever $m$ may be, hence showing the instability of a $\mathrm{CH}$ kink solution.

\subsection{1 order-one eigenvalues}

Multiplying (26) by $\varphi_{a}$, then averaging on $[0, L]$, we get: $\overline{\varphi_{a}, \mathcal{L}_{1} \varphi_{a}}=\sigma_{1} \overline{\varphi_{a}, \varphi_{a}}$. We can now deduce $\sigma_{1}$

$$
\sigma_{1}=-\frac{r t^{2}}{1+t^{2}-\frac{4}{s \Lambda}}+O\left(e^{-s \Lambda}\right), \quad t=\tan [\pi m / 2 n] .
$$

The order-one correction at $\sigma_{0}$ is only provided by friction and thus has a stabilizing effect on the inverse cascade generated by $\sigma_{0}$.

\subsection{2 order-two eigenvalues}

In the same way, we obtain an equation yielding $\sigma_{2}$

$$
\overline{\varphi_{a} \mathcal{L}_{1} \varphi_{a 1}}+\overline{\varphi_{a} \mathcal{L}_{2} \varphi_{a}}=\sigma_{1} \overline{\varphi_{a} \varphi_{a 1}}+\sigma_{2} \overline{\varphi_{a} \varphi_{a}}-\vartheta_{1} \overline{\varphi_{a} \varphi_{b_{1}}} .
$$

Using 26 and 28, and after considerable algebra, we can give an asymptotic value of $\sigma_{2}$

$$
\begin{aligned}
\sigma_{2}= & \frac{r^{2} \Lambda^{2} t^{2}}{96 s^{2} \lambda_{3}\left(1+t^{2}\right)^{2}}-\frac{\delta r^{2} G^{2}\left(4+9 t^{2}\right) t^{2} \Lambda^{4}}{276480 \lambda_{3} s^{2}\left(1+t^{2}\right)^{2}} \\
& -\frac{t^{2} G^{2}\left(4+3 t^{2}\right)}{12 R_{0}\left(1+t^{2}\right)}\left(\frac{\Lambda^{2}}{4}+\frac{\Lambda}{s\left(1+t^{2}\right)}-\frac{\pi^{2}}{s^{2}\left(4+3 t^{2}\right)}\right) \\
& +O\left(r^{2} \Lambda, G^{2} \delta P r^{2} \Lambda^{3}, G^{2} \Lambda^{-1}\right) .
\end{aligned}
$$

Stratification and dispersion therefore stabilize at the second order but are more efficient at large scales than friction. It is clear that dispersion prevails over the $G^{2}$ dissipative term provided that $\delta P r=O(1)$.

\subsubsection{Linear-stability criteria of the nonlinear stationary solution}

The condition of stability is given by $\sigma<0$ and yields in the case where $|\delta P r| \ll 1$

$$
\mathcal{G}_{c}^{2}=\frac{3}{2} s\left(\frac{w^{*}}{\Gamma}\right)^{3} e^{-s \frac{w^{*} \Lambda^{*}}{\Gamma}}\left(\frac{16}{\Lambda^{*}}\right)^{3},
$$

where $\Lambda^{*}=\Lambda / \epsilon$ is the period of the nonlinear wave in the frame defined with the fast $x$ coordinate. $\epsilon$ is expressed as a function of the maximum amplitude of the vertical velocity $w^{*}$ and $\Lambda^{*}$ : from $w^{*}=$ $\epsilon \Gamma \tanh s \Lambda / 4$, we have $\epsilon=w^{*} / \Gamma\left(1+O\left(e^{-s \Lambda^{*} w^{*} /(2 \Gamma)}\right)\right)$. The most stringent condition intervenes when $m=1$ and the true maximum is given by $m / n \rightarrow 0$. If $\delta P r=O(1)$, the stability condition yields

$$
\mathcal{G}_{c}^{2}=\frac{135 s}{8(\operatorname{Pr}-1)^{2}}\left(\frac{w^{*}}{\Gamma}\right)^{5} e^{-s \frac{w^{*}}{\Gamma} \Lambda^{*}}\left(\frac{16}{\Lambda^{*}}\right)^{5} .
$$

$\mathcal{G}_{c}^{2}$ is related to the overall Richardson number of the primary wave. $\mathcal{G}_{c}$ is very small, far smaller than $\mathcal{G}_{c}=0.363\left(w^{*} / \Gamma\right)^{3}$ given by the linear analysis and all the more so since the Prandtl number is different from one and the wavelength is very large. This structure is easily stabilized as numerical simulations previously showed for small values of stratification and dispersion. The weakly-modified $\mathrm{CH}$ assumption 
reveals to be very good. This theory shows however that dispersion has a greater stabilizing effect. When rotation is assumed, dispersion vanishes for $\mu=0$ so (32) is not in fact valid for near inertial frequencies. The effect of the slope is technically more difficult, highlighting the physical complexity, sometimes leading to chaos and we have decided to leave it for another paper.

We now compare the Prandtl number effect with rotation. In amplitude equations quoted in the subsection (4.3), solutions are stable numerically for weak rotation. In the contrary case, the initial evolution of a steady MKdV solution is strongly nonstationary and characterized by marked distortions of the wave shape. But due to the near-integrability of MKdV, after a time roughly equal to one wave period, the shape returns to its original form (Nikitenkova et al. (2000)). In a KdV, when dispersion due to rotation prevails over this due to stratification, periodic waves are possible. When both dispersions intervene, periodic waves have shapes which are combinations of parabola (domain where rotation is prevailing) and smooth pulses (the KdV solution). A perturbative method then enables one to give an analytical representation of these nonlinear waves with as small dimensionless parameter, the rotation dispersion. The same remarks can be made for the rotational modified Benjamin-Ono equation, i.e. waves long compared to one of the characteristic layers and short compared to the total depth. The effect of rotation on nonlinear internal waves generated by tides in the ocean was investigated by Gerkema (1996). Rotation affected these soliton-like nonlinear waves. The numerical solutions of these waves underwent a decrease of the number of solitons with rotation. The $\beta$ effect also decreases their number according to the negative-viscosity approach and the related inverse cascade. Our study shows as well that the dispersive action of the Prandtl number decreases the number of solitons. Dispersion therefore seems to have a similar effect in all these nonlinear waves: to increase the characteristic wavelength. So, we can expect the introduction of rotation to act together with the Prandtl number dispersive effect to decrease the number of solitons. Gerkema observed that rotation could even prevent the formation of solitons if it was strong enough. The possibility of wave breaking in the presence of rotational dispersion was also envisaged.

\section{Discussion}

We have investigated the linear destabilization of an internal wave and its eventual restabilization into a large-scale nonlinear wave. The assumption of a dissipative medium does not reduce the ability for an internal wave to destabilize. Not only are these waves still found unstable regardless of amplitude but if the Prandtl number is different from one, they are destabilized whatever the Reynolds number by a long-wavelength perturbation. Instability is driven by velocity shear when the Froude number is large or in the contrary case, by resonant interactions. The most unstable modes have the same periodicity as the base wave. A large-scale threshold is located close to the stablest point in the stability diagram defined by $P r=1$ and is characterized by very weak friction, inverse Froude number, incidence and Floquet parameter. If all these conditions hold, instability slightly departs from the Kolmogorov flow instability, at a Reynolds number $R_{c}$ above and near $\sqrt{2}$. An analytical representation of the marginal stability $R(\alpha)$ is possible in the neighbourhood of $\alpha=0$ whatever the Reynolds number. Although Rayleigh dissipation provides large critical Reynolds numbers in bounded and small-scale motions, it is not relevant in the geophysical context. Indeed, this friction strongly shifts $\alpha_{c}$ toward the small scales without greatly modifying $R_{c}$. On the contrary, the addition of a mean shear flow slowly varying with the vertical coordinate allows higher values of $R_{c}$ and $\mathcal{G}$. The generalization of this hydrodynamic-stability problem in the context of the wave-mean flow interaction is the subject of our current research.

For a slightly supercritical instability, the large-scale dynamics is modelled by an amplitude equation. Numerical solutions of this latter with the assumption of space periodicity show nonlinear waves whose neighbouring patterns interact and merge. The characteristic scale tends to increase while the linear wave feeds this inverse cascade. The new terms involved in the modified $\mathrm{CH}$ do not drive the cascade to a turbulent régime, in the same way as the previous studies of the Kolmogorov flow instability. A steady state is usually the final state of our simulations characterized by nonlinear waves travelling with a constant speed. Stratification delays and sometimes stops this process in a better way than friction before the gravest mode is attained. Incidence acts to accelerate this cascade when $|\mu|<1$ and leads to a chaotic state in a transitional stage when $\mu=O(1)$ and for small values of $G$; the coherent structure seems then not to exist over a reasonable duration of the simulations. Another kind of structure appears when $|\mu|>1$ of smaller period and amplitude. If the motion is related to a very-small-slope wave, $\varpi \ll 1$, the disturbance then remains weak and the primary wave is well preserved otherwise the disturbance 
blows up. By omitting turbulence generation, our approach here fails to describe the saturation and the possible wave breakdown. Dispersion accelerates the cascade more weakly than incidence or the $\beta$-effect because of the competing action of stratification but may arrest it. The dispersive effect of the Prandtl number has not been yet reported to the best of our knowledge.

An approximate stationary solution of the amplitude equation can be given in the context of a weakly modified Cahn-Hilliard equation and when the scale attained by the cascade is very large. The stability analysis of this solution shows as does the numerical study how easily weak stratification and dispersion can yield long-lived structures and also demonstrates the prevailing stabilizing rôle of dispersion over stratification, as well as the possibility of a nonlinear stabilization of the stationary linear wave at a far larger Froude number than linear analysis had predicted. When a weakly rotating flow is considered, dispersion only acts when the slope is non-zero and the stabilizing dispersion effect is balanced by the quadratic destabilizing slope effect. A careful analysis of the slope should therefore be undertaken. For a stronger rotation, the nonlinear wave existence is not assured. According to the literature, the assumption of a three-dimensional weakly rotating flow would not change these results. On the contrary, a weak rotation would accelerate the inverse cascade.

These nonlinear waves can transport their energy vertically in a more efficient way than the primary wave and in the same direction as the wave phase in spite of the absence of turbulence. This is all the more significant as low-frequency waves are very energetic and as high-frequency waves are believed to accomplish most of the energy transport in the atmosphere (Fritts and Rastogi (1985)).

Stratification constrains horizontal and above all vertical motions. The horizontal-scale reduction was highlighted here; our model reveals its weaknesses since we have omitted to add a vertical scaling to model the layering effect. Despite low Reynolds and overall Richardson numbers, this work can however be related to previous studies of stability of internal waves and stratified shear flows. Klaassen and Peltier (1991) numerically analysed the instability of a KH billow in a very weakly stratified flow with $\operatorname{Pr}=1$. This large-Reynolds-number instability was also shear driven. Smyth (2003) made the same conclusion after examining the latter problem while varying $\operatorname{Pr} \geq 1$. He observed moreover, that increasing $\operatorname{Pr}$ increased the tendency towards instability. Thorpe (1994) forced an overturned internal wave in a horizontal tube filled with a stratified fluid. Our comparison can be only qualitative: experimental Prandtl numbers are high $(\operatorname{Pr} \sim 700)$ and the Richardson number is of order unity. Nevertheless, secondary Kelvin-Helmoltz-like instabilities generated nonlinear waves and Thorpe pointed out the rôle of the Prandtl number on these processes.

We thank B. Legras and V. Tseitline at the Laboratoire de Météorologie Dynamique, CNRS, Paris for the suggestion of this study, comments and criticisms. We also acknowledge R. Grimshaw and S. Maslowe for helpful discussions and corrections of the proof. The author would like to thank both the anonymous referees for their numerous technical advice and references.

\section{A Long-wave marginal stability}

We give the expressions of the Reynolds number of a neutral mode as a function of the different parameters of the flow and the rescaled wavenumber $K$.

function of the Prandtl number:

$$
\begin{aligned}
R= & \sqrt{2} \frac{[D(K)]^{\frac{1}{2}}}{K^{2}}+\mathcal{G}[D(K)]^{\frac{1}{2}} \frac{4 K^{16}-4 D(K)+2 K^{12} D(K)+K^{8}[7 D(K)-2]}{2 \sqrt{2} K^{8}\left[K^{8}+D(K)\right]} \\
& -(P r-1) \frac{4 K^{16}+12 D(K)-4 K^{12} D(K)+7 K^{8} D(K)+6 K^{8}}{4 \sqrt{2} K^{10}\left[K^{8}+D(K)\right]} \\
& -\frac{(P r-1)^{2}}{\mathcal{G}}[D(K)]^{\frac{1}{2}} \frac{4 D(K)+K^{8}[2+D(K)]}{4 \sqrt{2} K^{12}\left[K^{8}+D(K)\right]}+O\left(\mathcal{G}^{2}\right),
\end{aligned}
$$

with $D(K)=1+\left(1+K^{8}\right)^{1 / 2}$.

function of the slope and the friction:

$$
R^{(0)}=\left(1+\varpi^{2}\right)^{\frac{1}{2}} \frac{\left(E(K)+\left[E(K)^{2}+4 K^{6}\left(1-7 \varpi^{2}\right)\left(F+K^{2}\right)\left(1+\varpi^{2}\right)\right]^{\frac{1}{2}}\right)^{\frac{1}{2}}}{\left(1-7 \varpi^{2}\right)^{\frac{1}{2}} K^{2}},
$$


with $\eta=F \mathcal{G}$ and $E(K)=2\left(1+\varpi^{2}\right)^{2}+F K^{2}\left(1+\varpi^{2}\right)+8 K^{4} \varpi^{2}$.

The conditions of such an expansion are: $\eta=O(\mathcal{G})$ and $\varpi$ must be less than $7^{-1 / 2}$.

\section{B Derivation of the amplitude equation}

We restrict ourselves to $\phi$-periodic solutions. By $\phi$-averaging the equations of motion, the two following conditions appear at each order,

$$
\begin{array}{r}
\epsilon^{6} \partial_{T X^{2}}\langle\psi\rangle+2 \epsilon^{2} \varpi\left\langle\partial_{X^{2} \phi} \psi \partial_{\phi} \psi\right\rangle+\epsilon^{3}\left\langle\partial_{X}^{3} \psi \partial_{\phi} \psi\right\rangle \\
-\epsilon^{3}\left\langle\partial_{X}^{3} \psi \sin \phi\right\rangle-2 \epsilon^{2} \varpi\left\langle\partial_{X^{2} \phi} \psi \sin \phi\right\rangle-\epsilon^{3}\left\langle\partial_{X^{2} \phi} \psi \partial_{X} \psi\right\rangle \\
-2 \epsilon^{2} \varpi\left\langle\partial_{X \phi^{2}} \psi \partial_{X} \psi\right\rangle=-\epsilon \mathcal{G} \partial_{X}\langle\xi\rangle+\frac{1-\epsilon^{2}}{R_{0}} \epsilon^{4} \partial_{X}^{4}\langle\psi\rangle-\eta \frac{1-\epsilon^{2}}{R_{0}} \epsilon^{2} \partial_{X}^{2}\langle\psi\rangle, \\
\epsilon^{4} \partial_{T}\langle\xi\rangle+\epsilon\left\langle\partial_{X} \xi \partial_{\phi} \psi\right\rangle-\epsilon\left\langle\partial_{\phi} \xi \partial_{X} \psi\right\rangle \\
-\epsilon\left\langle\partial_{X} \xi \sin \phi\right\rangle-\epsilon\left(1+\varpi^{2}\right)^{\frac{1}{2}}\left\langle\partial_{X} \psi \sin \phi\right\rangle=\epsilon \mathcal{G} \partial_{X}\langle\psi\rangle+\frac{1-\epsilon^{2}}{S_{0}} \epsilon^{2} \partial_{X}^{2}\langle\xi\rangle .
\end{array}
$$

The successive solvability conditions prescribed by both these equations are:

Order 0: $\psi^{(0)}$ and $\xi^{(0)}$ are only functions of $X$ and $T$.

Order $\epsilon$ : equation (B2) leads to what $\mathcal{G}$ is at the most of order $\epsilon$.

Order $\epsilon^{2}$ : equation (B1) yields $\mathcal{G}=O\left(\epsilon^{2}\right)$, equation (B2) gives the relationship

$$
\partial_{X}^{2} \xi^{(0)}=\frac{S_{0}}{2} \frac{R_{0}-S_{0}}{\left(1+\varpi^{2}\right)^{\frac{1}{2}}} \partial_{X}^{2} \psi^{(0)} .
$$

Order $\epsilon^{3}$ : equation (B1) leads to $\mathcal{G}=O\left(\epsilon^{3}\right)$ and equation (B2), $\left\langle\xi^{(1)}\right\rangle=0$.

Order $\epsilon^{4}$ : equation (B1) gives

$$
-\frac{\varpi R_{0}^{2}}{\left(1+\varpi^{2}\right)^{2}}\left[\partial_{X} \psi^{(0)} \partial_{X}^{3} \psi^{(0)}+\left(\partial_{X}^{2} \psi^{(0)}\right)^{2}\right]+\left[\frac{R_{0}}{2\left(1+\varpi^{2}\right)}-\frac{1}{R_{0}}-\frac{4 \varpi^{2} R_{0}}{\left(1+\varpi^{2}\right)^{2}}\right] \partial_{X}^{4} \psi^{(0)}=-G \partial_{X} \xi^{(0)} .
$$

By combining with the preceding relation, we obtain

$$
\frac{R_{0}}{2}-\frac{1}{R_{0}}=0, \quad \varpi=O(\epsilon), \quad R_{0}=S_{0}, \quad \xi^{(0)}=0 .
$$

Equation (B2) induces the relationship (12) connecting $\left\langle\xi^{(2)}\right\rangle$ and $\psi^{(0)}$.

Order $\epsilon^{5}$ : equation (B1) yields $\varpi=O\left(\epsilon^{2}\right)$ and $\delta P r=O\left(\epsilon^{2}\right)$.

Order $\epsilon^{6}$ : equation (B1) gives the scaling for the friction $\eta=O\left(\epsilon^{4}\right)$ and yields the second relationship (13) linking $\left\langle\xi^{(2)}\right\rangle$ and $\psi^{(0)}$.

\section{Vertical energy flux $\langle\overline{P v}\rangle$}

The pressure is obtained straightforwardly from the horizontal momentum equation, multiplying it by the vertical velocity and phase-averaging yield

$$
\langle P v\rangle=-\epsilon^{3} \partial_{X} \psi^{(0)^{3}}-3 \epsilon^{4} \partial_{X} \psi^{(0)^{2}} \partial_{X}\left\langle\psi^{(1)}\right\rangle+O\left(\epsilon^{5}\right)=\langle v\rangle^{3}+O\left(\epsilon^{5}\right) .
$$

Using (12) and (13), we can derive the expression of $\int \partial_{X} \psi^{(0)^{3}} d x$. Under the assumption of periodic boundary conditions for $\partial_{X}^{-2} \psi^{(0)}$, we have :

$$
\begin{aligned}
\int v_{0} \partial_{T} \psi^{(0)} d X=-\frac{3}{2} R_{0} \int \partial_{X} v_{0} \partial_{X}^{2} v_{0} d X & -r \int \psi^{(0)} v_{0} d X+\frac{1}{2} G \delta P r \int \psi^{(0)^{2}} d X \\
& +G \int v_{0}^{2} d X+\mu \int v_{0}^{3} d X+\frac{G^{2}}{2 R_{0}} \int \partial_{X}\left[\left(\partial_{X}^{-1} \psi^{(0)}\right)^{2}\right] d X
\end{aligned}
$$

with $v_{0}=-\partial_{X} \psi^{(0)}$. So, extracting $\int v_{0}^{3} d X$, we get

$$
\overline{v_{0}^{3}}=\frac{1}{\mu}\left(\overline{v_{0} \partial_{T} \psi^{(0)}}-G \overline{v_{0}^{2}}-\frac{1}{2} G \delta \operatorname{Pr} \overline{\psi^{(0)^{2}}}\right) \text {. }
$$




\section{References}

Afanasyev, Y. and Peltier, W. (2001). Numerical simulations of internal gravity wave breaking in the middle atmosphere: the influence of dispersion and three-dimensionalization. J. Atmos. Sci., 58:132153.

Balmforth, N. and Young, Y. (2002). Stratified Kolmogorov flow. J. Fluid Mech., 450:131-167.

Batchayev, A. M. and Kurganskiy, M. V. (1986). Periodic shear flow instability in a weakly stratified fluid. Izvestiya, Atmospheric and Oceanic Physics, 22(1):1-5.

Caillol, P. (2000). Quelques effets des nonlinéarités sur l'existence des structures cohérentes rencontrées en Géophysique, Université Pierre et Marie Curie, Paris. PhD thesis.

Caillol, P. and Zeitlin, V. (2000). Kinetic equations and stationary energy spectra of weakly nonlinear internal gravity waves. Dyn. of Atmos. and Oceans, 32:81-112.

Campbell, L. J. and Maslowe, S. A. (2003). Nonlinear critical-layer evolution of a forced gravity wave packet. J. Fluid Mech., 493:151-179.

Dewan, E. M. and Good, R. E. (1986). Saturation and the "universal" spectrum for vertical profiles of horizontal scalar winds in the atmosphere. J. Geophys. Res., 91:2742-2748.

Drazin, P. (1977). On the instability of an internal gravity wave. Proc. R. Soc. Lond. A., 356:411-432.

Frisch, U., Villone, B., and Legras, B. (1996). Large-scale Kolmogorov flow on the $\beta$ plane and resonant wave interactions. Physica D, 94:36-56.

Fritts, D., Tsuda, T., Sato, T., Fukao, S., and Kato, S. (1988a). Observational evidence of a saturated gravity-wave spectrum in the troposphere and lower stratosphere. J. Atmos. Sci., 45(12):1741-1759.

Fritts, D. C. (1984). Shear excitation of atmospheric gravity waves. part ii: Nonlinear radiation from a free shear layer. J. Atmos. Sci., 41(4):524-537.

Fritts, D. C. and Rastogi, P. (1985). Convective and dynamical instabilities due to gravity motions in the lower and middle atmosphere: Theory and observations. Radio Sci., 20:1247-1277.

Fritts, D. C., Smith, S. A., Balsley, B. B., and Philbrick, C. R. (1988b). Evidence of gravity wave saturation and local turbulence production in the summer mesosphere and lower thermosphere during the state experiment. J. Geophys. Res., 93:7015-7025.

Grimshaw, R., Ostrovsky, L., Shrira, V., and Stepanyants, Y. (1998). Long nonlinear surface and internal gravity waves in a rotating ocean. Survey in Geophys., 19:289-338.

Hines, C. (1963). The upper atmosphere in motion. Quart. J. Roy. Meteor. Soc., 89(379):1-42.

Huang, H. P. and Robinson, A. (1998). Two-dimensional turbulence and persistent zonal jets in a global barotropic model. J. Atmos. Sci., 55:611-632.

Kato, S. (1980). In Dynamics of the upper Atmosphere. D. Reidel Publishing Company/Dordrecht.BostonLondon.

Kawasaki, K. and Ohta, T. (1982). Kink dynamics in one-dimensional nonlinear systems. Physica A, 116(3):573-593.

Khapaev, A. (2000). Stability of internal gravity waves propagating at small angles to the vertical. Izvestiya, Atmosph. and Oceanic Phys., 36(1):57-63.

Klaassen, G. and Peltier, W. (1985). The onset of turbulence in finite-amplitude Kelvin-Helmholtz billows. J. Fluid Mech., 155:1-35.

Klaassen, G. and Peltier, W. (1991). The influence of stratification on secondary instability in free shear layers. J. Fluid Mech., 227:71-106. 
Klostermeyer, J. (1991). Two- and three-dimensional parametric instabilities in finite-amplitude internal gravity waves. Geophys. Astrophys. Fluid Dyn., 61:1-25.

Kurganskiy, M. (1980). Instability of internal gravity waves propagating at small angles to the vertical. Izvestiya, Atmospheric and Oceanic Physics, 16(10):758-764.

Lamb, H. (1945). In Hydrodynamics. Dover Publications, New-York (1879).

Legras, B. and Villone, B. (2003). Dispersive and friction-induced stabilization of an inverse cascade. the theory for the Kolmogorov flow in the slightly supercritical regime. Physica D, 175:139-166.

Legras, B., Villone, B., and Frisch, U. (1999). Dispersive stabilization of the inverse cascade for the Kolmogorov flow. Phys. Rev. Lett., 82:4440-4443.

Lelong, M. and Dunkerton, T. (1998). Inertia-gravity wave breaking in three dimensions. part i: convectively stable waves. part ii: convectively unstable waves. J. Atmos. Sci., 55:2473-2501.

Lombard, P. N. and Riley, J. J. (1996). Instability and breakdown of internal gravity waves. i. linear stability analysis. Phys. Fluids, 8(12):3271-3287.

Manfroi, A. and Young, W. (1999). Slow evolution of zonal jets on the beta-plane. J. Atmos. Sci., $56: 784-800$.

Meek, C., Reid, I., and Manson, A. (1985). Observations of mesospheric wind velocities. i: Gravity wave horizontal scales and phase velocities determined from spaced wind observations. Radio Sci., 20:1363-1382.

Miyazaki, T. and Adachi, K. (1998). Short-wavelength instabilities of waves in rotating stratified fluids. Phys. of Fluids, 10(12):3168-3177.

Nikitenkova, S. P., Stepanyants, Y. A., and Chikhladze, L. M. (2000). Solutions of the modified Ostrovskiy equation with cubic non-linearity. J. Appl. Maths. Mechs., 64(2):267-274.

Prusa, J., Smolarkiewicz, P., and Garcia, R. (1996). Propagation and breaking at high altitudes of gravity waves excited by tropospheric forcing. J. Atmos. Sci., 53(15):2186-2216.

She, Z. (1987). Metastability and vortex pairing in the Kolmogorov flow. Phys. Lett. A, 124(3):161-164.

Sivashinsky, G. (1985). Weak turbulence in periodic flows. Physica D, pages 243-255.

Smyth, W. D. (2003). Secondary Kelvin-Helmholtz instability in weakly stratified shear flow. J. Fluid Mech., 497:67-98.

Sutherland, B. (2001). Finite-amplitude internal wavepacket dispersion and breaking. J. Fluid Mech., 429:343-380.

Thorpe, S. A. (1994). Statically unstable layers produced by overturning internal gravity waves. IMA Jour. of Appl. Math., 260:333-350.

Tsuda, T., Kato, S., Nakamura, T., Vincent, R., Manson, A., C.E., M., and R.L., W. (1994). Variations of the gravity wave characteristics with height, season and latitude revealed by comparative observations. J. Atmos. Sol. Terr. Phys., 56:555-568.

Tsuda, T., Van Zandt, T., M., M., Kato, S., and Fukao, S. (1991). Spectral analysis of temperature and Brunt-Vaisala frequency fluctuations observed by radiosondes. J. Geophys. Res., 96(D2):17265-17278.

Walterscheid, R. L. and Boucher, D. J. (1984). A simple model of the transient response of the thermosphere to impulsive forcing. J. Atmos. Sci., 41(6):1062-1072.

Weinstock, J. (1990). Saturated and unsaturated spectra of gravity waves and scale-dependent diffusion. J. Atmos. Sci., 47:2221-2225.

Winters, K. B. and D'Asaro, E. A. (1994). Three-dimensional wave instability near a critical level. J. Fluid Mech., 272:255-284. 
Wu, Y. and Widdel, H. U. (1991). Further study of a saturated gravity wave spectrum in the mesosphere. J. Geophys. Res., 96(D5):9263-9272.

Yi, F. (1999). Resonant interactions between propagating gravity wave packets. J. Atmos. Sol. Terr. Phys., 61:675-691.

Young, Y. (1999). On stratified Kolmogorov flow. Woods Hole Oceanogr. Inst. Tech. Rep. 40.

Zhang, S. D. and Yi, F. (2002). A numerical study of propagation characteristics of gravity wave packets propagating in a dissipative atmosphere. J. Geophys. Res., D14:4222-4230.

Zhang, S. D., Yi, F., and Wang, J. F. (2000). The nonlinear effects on the characteristics of gravity wave packets: dispersion and polarization relations. Ann. Geophys., 18:1316-1324. 
Figure 1: Stability boundaries in the $(\alpha, R)$ plane of an internal wave with $\operatorname{Pr}=1$ : (a) for different stratifications and $\varpi=0$. From curves 1 to $8, \mathcal{G}$ is respectively, 0, 0.005, 0.01, 0.1, 0.2, 0.6, 1 and 1.05 . When $\mathcal{G}=0.6$, the two lobes in I reconnect at $R \simeq 30$. The third lobe reconnects with the second at $R \simeq 22$; (b) for different incidences and $\mathcal{G}=0.1$. $-\varpi=0, \cdots \varpi=0.2,--\varpi=0.4,-\cdot-\varpi=0.7$, $-\cdots \varpi=1$ and $--\varpi=2$.

Figure 2: Stability boundaries in the $(\alpha, R)$ plane of an internal wave for different $\eta$ : (a) unstratified case, $-\eta=0, \cdots \eta=0.005,--\eta=0.01,-\cdot-\eta=0.05$ and $--\eta=0.5 ;$ (b) $\mathcal{G}=0.05,-\eta=0$, $\cdots \eta=0.02,--\eta=0.05,-\cdots-\eta=0.2,-\cdots \eta=0.5$ and $--\eta=1$. Note the appearance of a small bump at $\eta=1$ in the $\alpha$-range where stratification acts the most efficiently. The curve for $\eta=0.05$ joins the frictionless marginal curve at $R \simeq 20$. Crosses indicate the minima given by (11) with $R=R^{(0)}+\mathcal{G}^{2} R^{(2)}$.

Figure 3: Stability boundaries in the $(\alpha, R)$ plane of an internal wave for different $\operatorname{Pr}$ and $\varpi=0$ : (a) Two stratification levels, $\mathcal{G}=0.2,-\operatorname{Pr}=1, \cdots \operatorname{Pr}=1.2 ; \mathcal{G}=0.6,--\operatorname{Pr}=1,-\quad-\operatorname{Pr}=5 ;(\mathrm{b})$ $\mathcal{G}=0.1, \cdots \operatorname{Pr}=0$ (curve 1 in figure $1(\mathrm{a})$ ), $--\operatorname{Pr}=0.1,-\cdots \operatorname{Pr}=0.8,--\operatorname{Pr}=1, \cdots \operatorname{Pr}=1.05$, $--\operatorname{Pr}=2,-\cdot-\operatorname{Pr}=4$ and $-\operatorname{Pr}=100$. Other negative-growth-rate tongues appear near $\alpha=0$, but for more clarity, we have only shown the widest one.

Figure 4: Stability boundaries in the $(\alpha, R)$ plane of an internal wave for different $\operatorname{Pr}$ with $\mathcal{G}=0.1$ : (a) $\varpi=0, \cdots \operatorname{Pr}=0,-\cdots \operatorname{Pr}=0.8,-\operatorname{Pr}=1, \cdots \operatorname{Pr}=1.05,--\operatorname{Pr}=4,-\quad-\operatorname{Pr}=50$ and $-\cdots \operatorname{Pr}=100$, in II, $R_{c} \simeq 65$ for $\operatorname{Pr}=1$ and $R_{c} \simeq 10.3$ for $\operatorname{Pr}=100$; (b) $\varpi=1,-\operatorname{Pr}=0.5$, $\cdots \operatorname{Pr}=1,--\operatorname{Pr}=2$.

Table 1: Maximum values of the inverse Froude number allowing an instability at finite $\alpha_{c}$. Friction does not act on $\mathcal{G}_{c}$ when the wave has a slope. Varying $\operatorname{Pr}$ does not change the results.

Figure 5: (a) Numerical (points) and asymptotic (lines) solutions of marginal stability $(\alpha, R):(\mathrm{a}) \star,-$, $\cdots \mathcal{G}=0.01 ;+,-\cdots,--\mathcal{G}=0.05, \eta=0.02 ; \diamond,--,-\cdot-\mathcal{G}=0.1$. The best fit is given by the inner expansion. (b) Location of the critical points $\left(\alpha_{c}, R_{c}\right)$ as functions of $\gamma$ : $-\mathcal{G}=0.01, \cdots \mathcal{G}=0.05,--$ $\mathcal{G}=0.1$ and $\diamond$, points obtained by the numerical analysis for $0 \leq \mathcal{G} \leq 0.2$.

Figure 6: Vertical velocity $--w$ and buoyancy $\cdots \xi$ as functions of $x$ at a given time, energy distribution following the modes as a function of time: $-\alpha=1, \cdots \alpha=2,--\alpha=3,-\cdot-\alpha=4$ and $-\cdots \alpha=5$; (a) \& (b) $\mathrm{CH} ;$ (c) \& (d) $G=10^{-2}$.

Figure 7: Vertical velocity and buoyancy as functions of $x$, energy distribution as a function of time, $G=10^{-3}, \delta P r=1:$ (a) \& (b) $\mu=0 ;$ (c) \& (d) $\mu=0.01$; same legend as in 6 .

Figure 8: Energy distribution as a function of time: $G=10^{-3}$, (a) $\delta P r=10$; (b) $\delta P r=1$ and $\mu=0.1$; (c) $\delta P r=1$ and $\mu=0.5$; same legend as in 6; (d) Vertical velocity and buoyancy as functions of $x \delta P r=1$ and $\mu=1$. 
Figure 9: Streamlines in the vertical plane (coordinates in multiples of $2 \pi: p=10$ ). The scale is not respected; the horizontal period is about 28.6 times the vertical wavelength, $R \simeq 1.587, S \simeq 1.782$ and $\mathcal{G} \simeq 8.610^{-5}(F r=1 / G, \epsilon=0.35):$ (a) the initial wave is stationary; (b) the initial wave has a slope: $\varpi=1.225 \%$; the structures with a horizontal period $\sim 5$ are modulated by the phase whose period is near 29 , as result, the vortex cores are tilted; (c) $\varpi=12.25 \%$ no periodicity is noticeable; (d) $\varpi=4 \%$, the nonlinear wave has a period $\sim 1.7$, the phase period is $\sim 2.5$, the combination of both yields a period $1 /(1 / 1.7-1 / 2.5) \sim 5(\epsilon=0.1)$.

Figure 10: $G=210^{-3}, \delta P r=0$ and $\mu=4$; (a) Vertical velocity and buoyancy as functions of $x$, note the lower amplitudes; (b) Energy distribution: the mode which has the largest amount of energy is 6. 


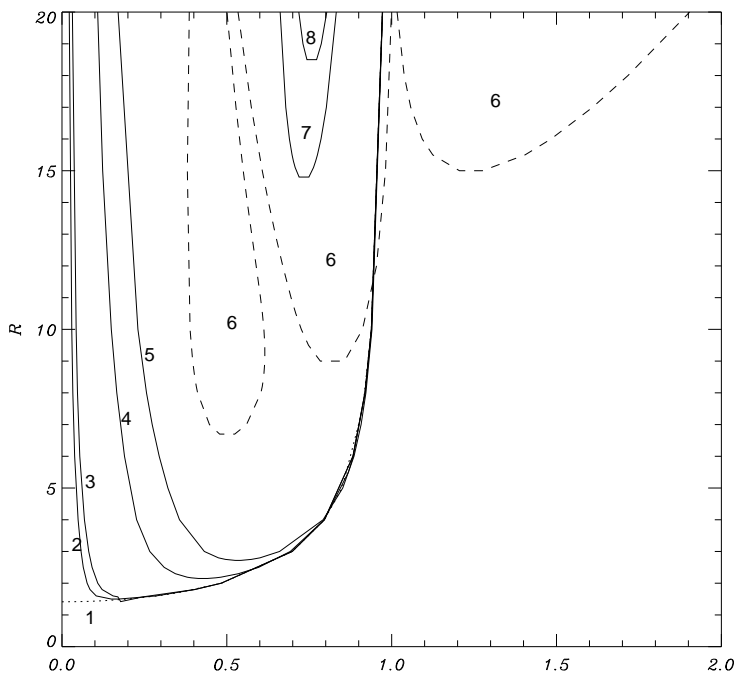

a)

a)

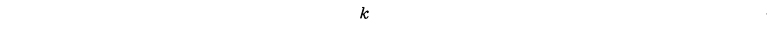

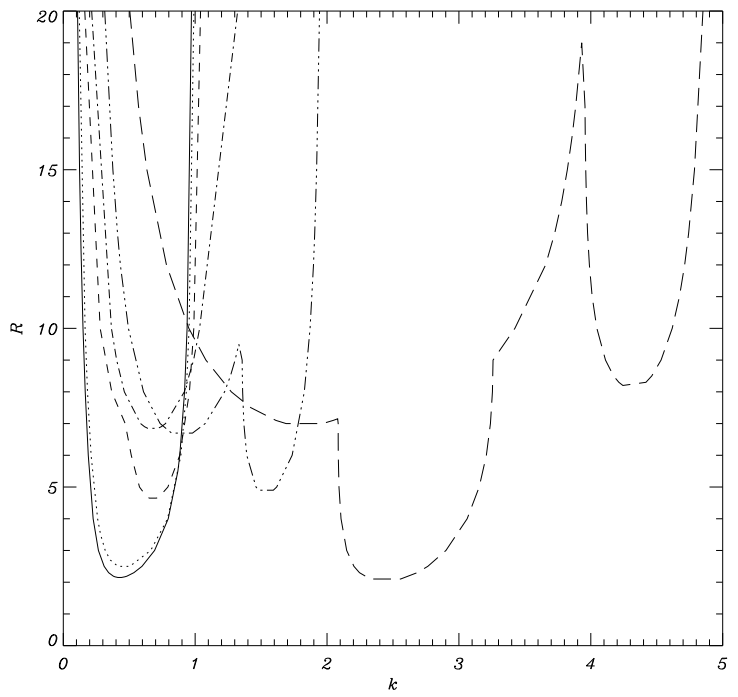

b)

Figure 1:

\begin{tabular}{|c|c|c|c|}
\hline domain & $\varpi=0, \eta=0$ & $\varpi=0, \eta \neq 0$ & $\varpi \neq 0$ \\
\hline I & $\mathcal{G}_{c}=1.2$ & $\mathcal{G}_{c}<1.2$ & no boundary \\
& & $\eta / R=0.1, \mathcal{G}_{c}=0.999$ & \\
\hline II & $\mathcal{G}_{c}=1$ & $\mathcal{G}_{c}<1$ & no boundary \\
& & $\eta / R=0.1, \mathcal{G}_{c}=0.994$ & \\
\hline
\end{tabular}

Table 1:

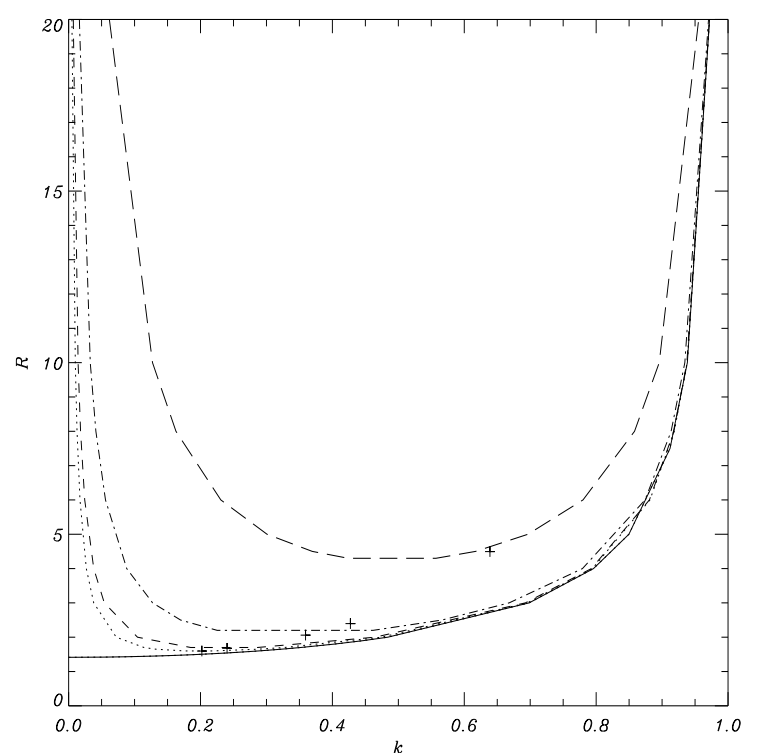

a)

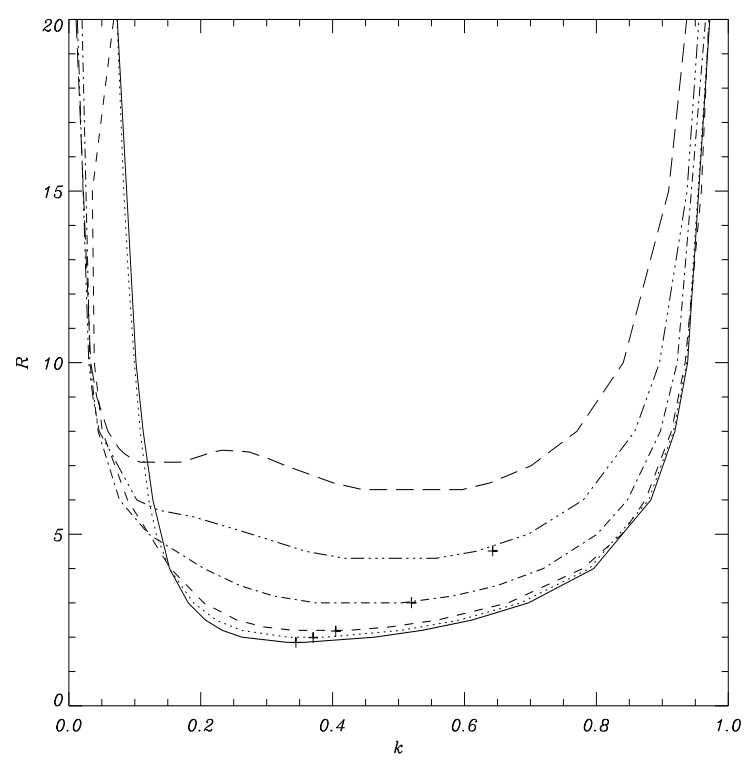

b)

Figure 2: 
(a)

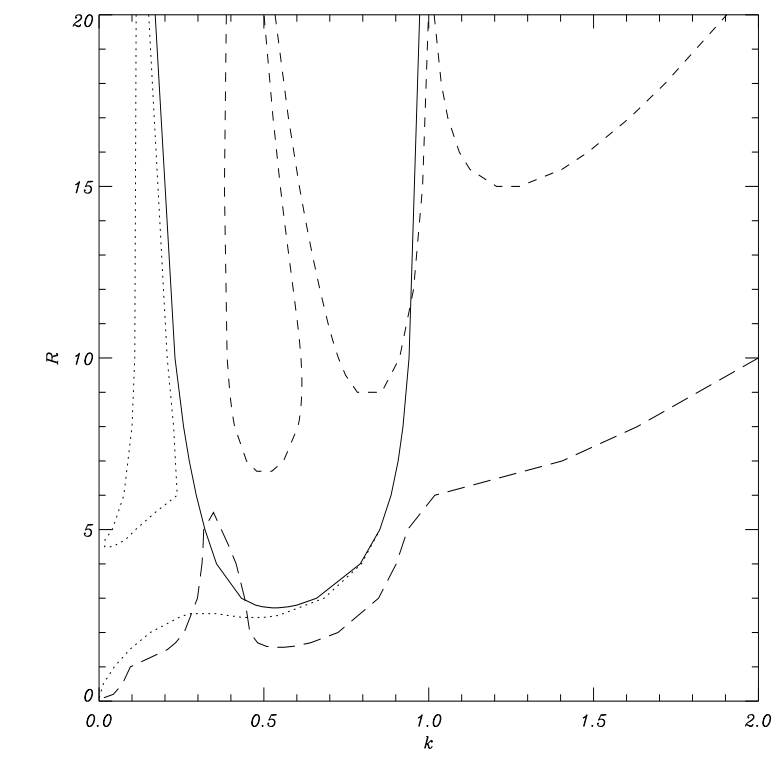

(b)

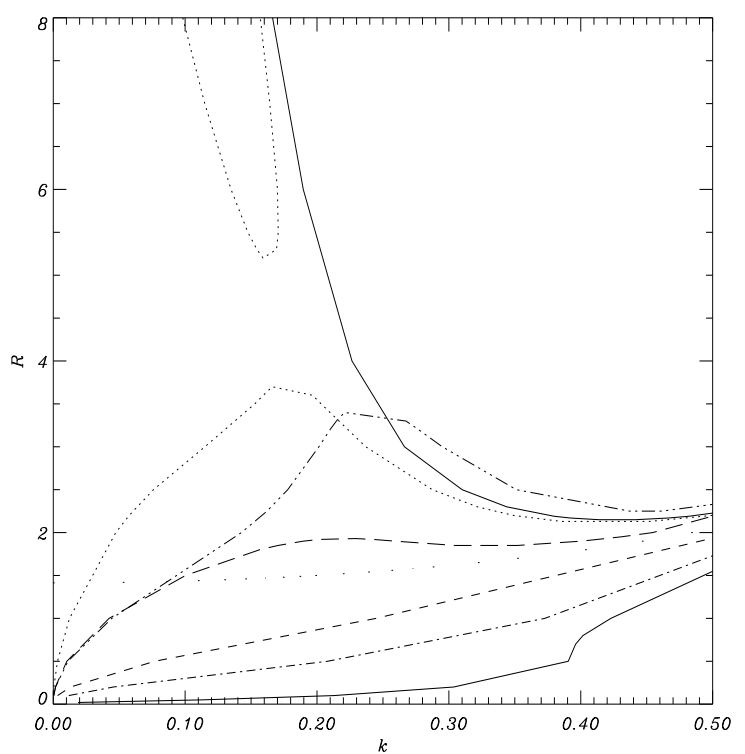

Figure 3:

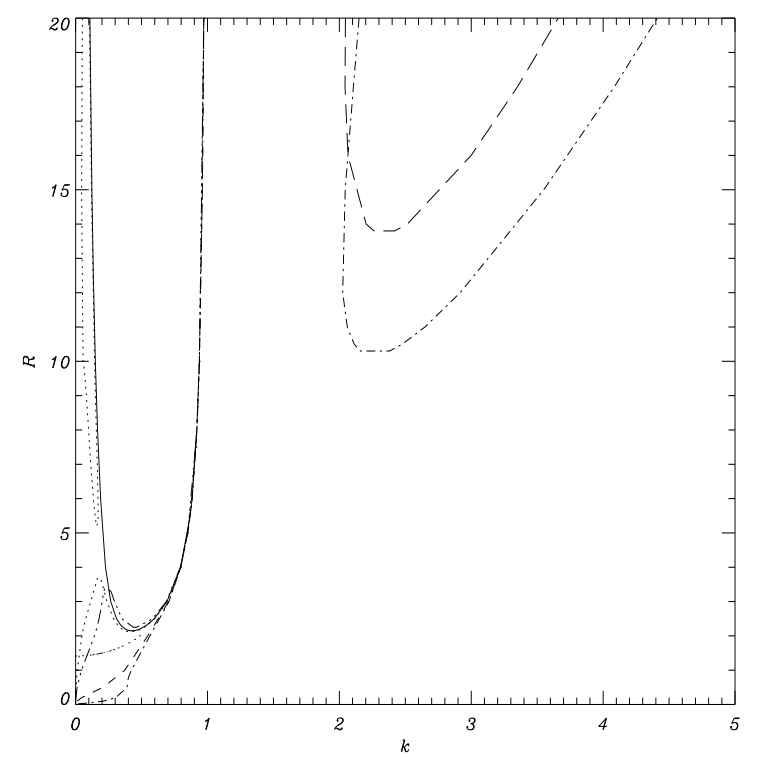

(a)

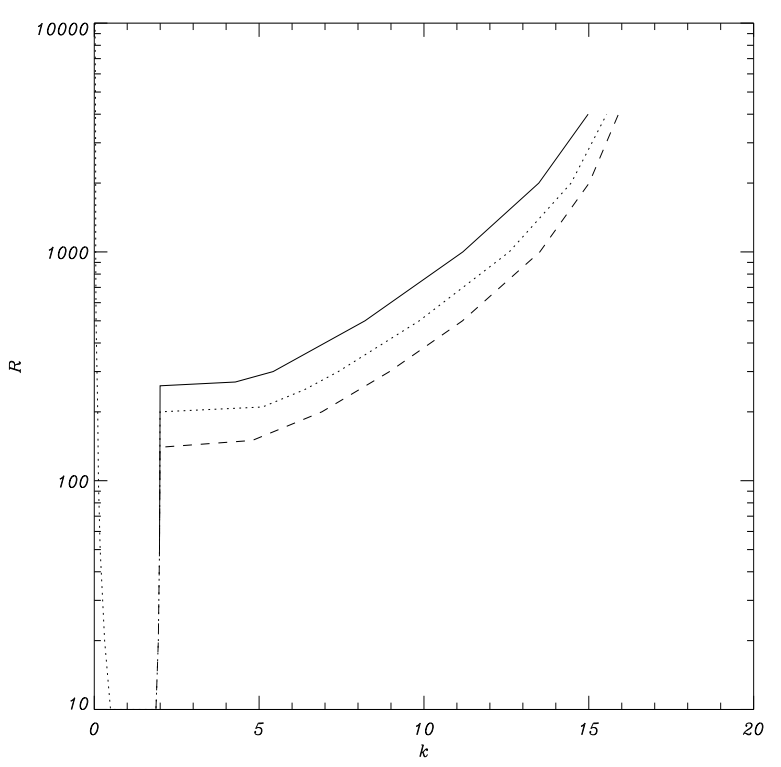

Figure 4: 
(a)
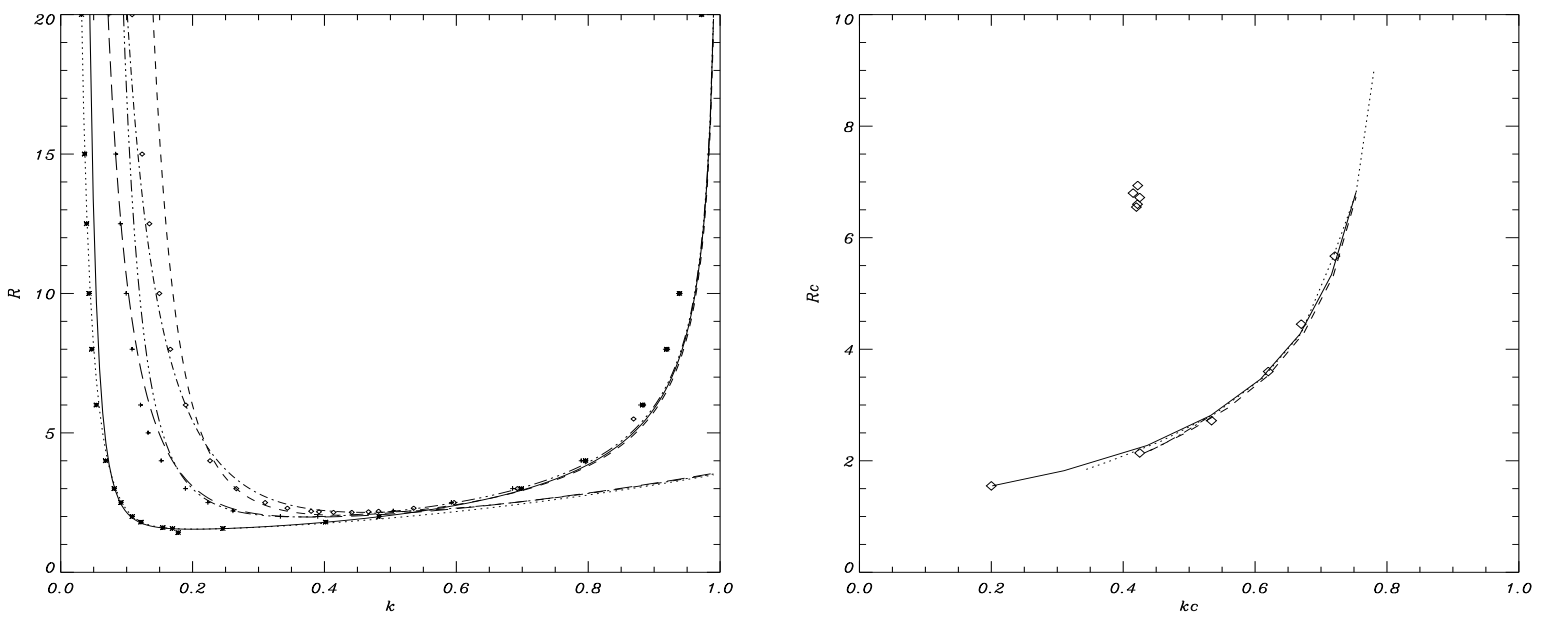

Figure 5:

(a)

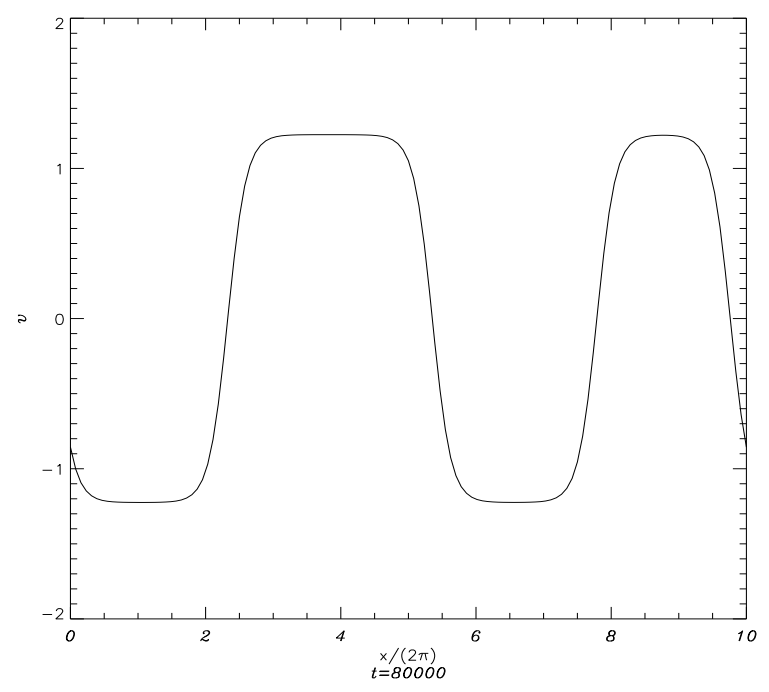

(c)

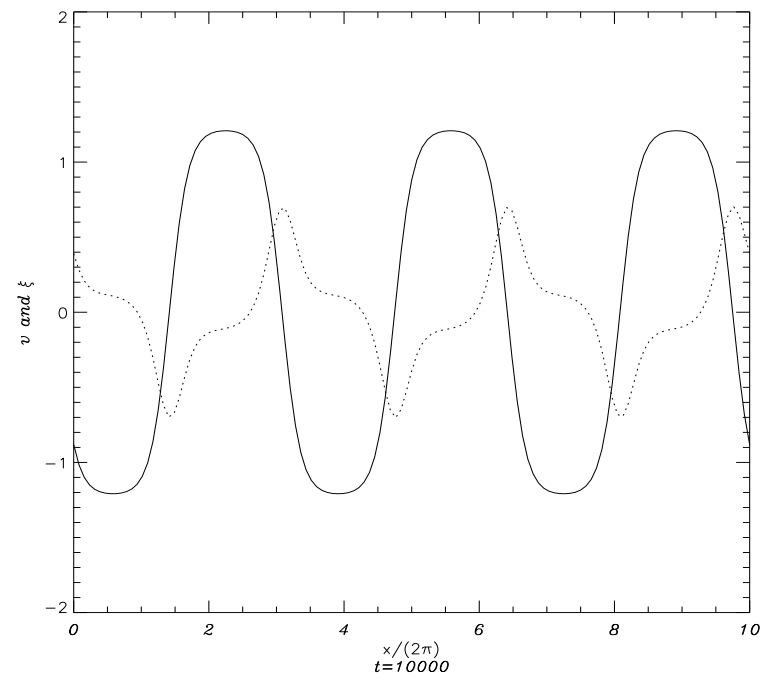

(b)
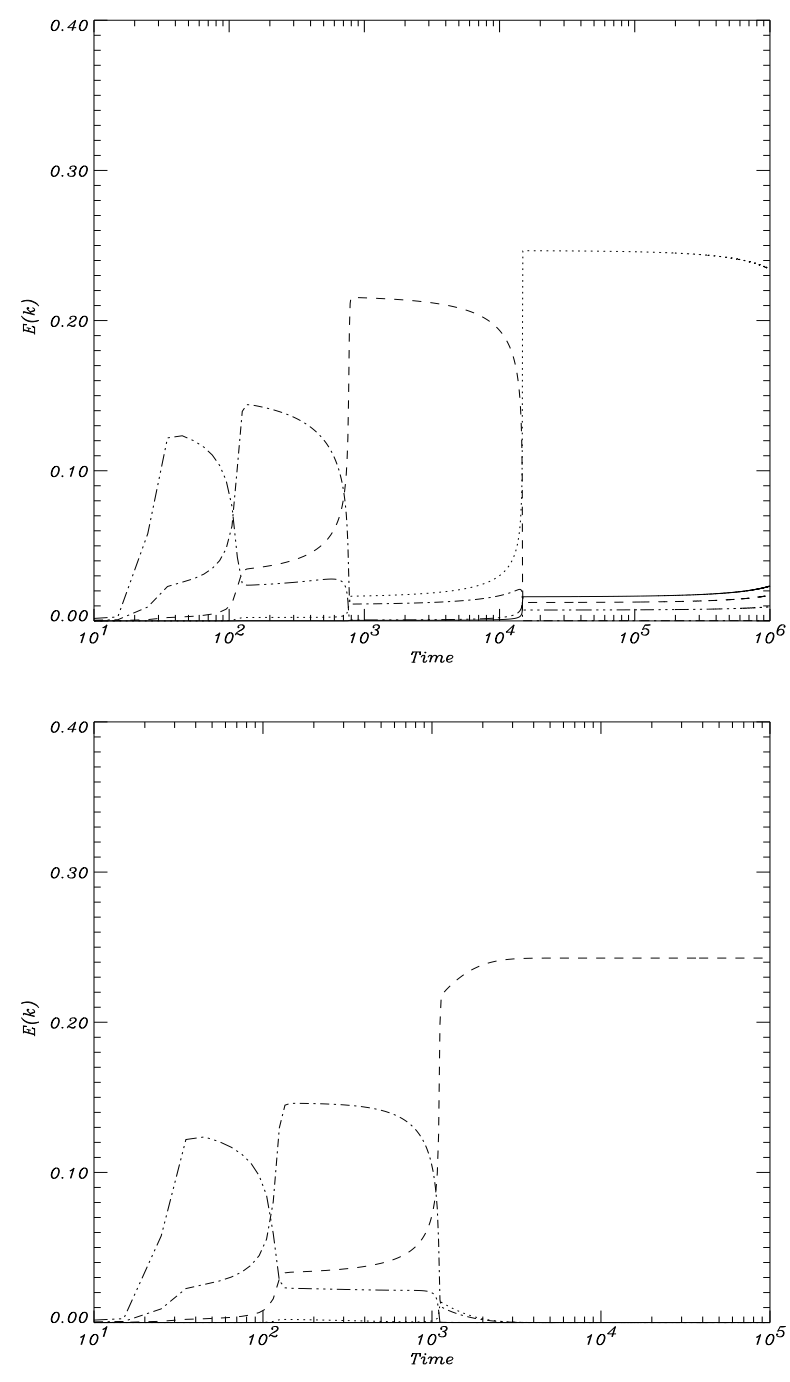

Figure 6: 
(a)

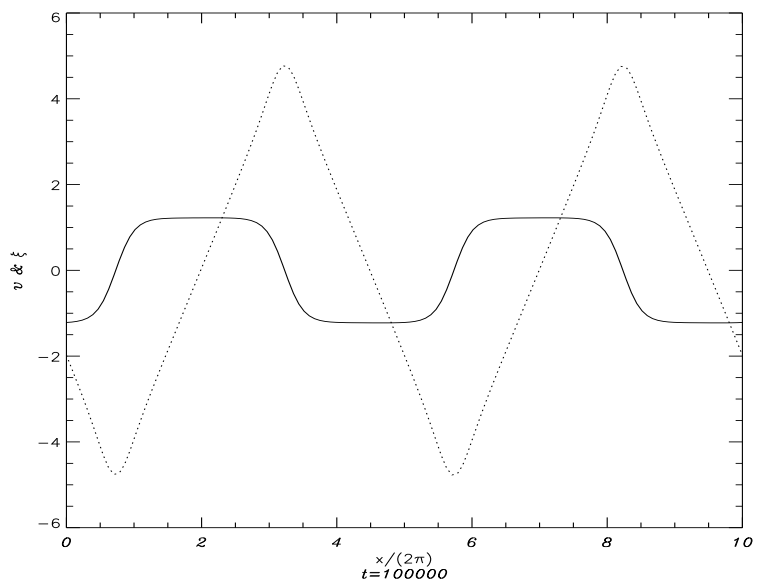

(c)

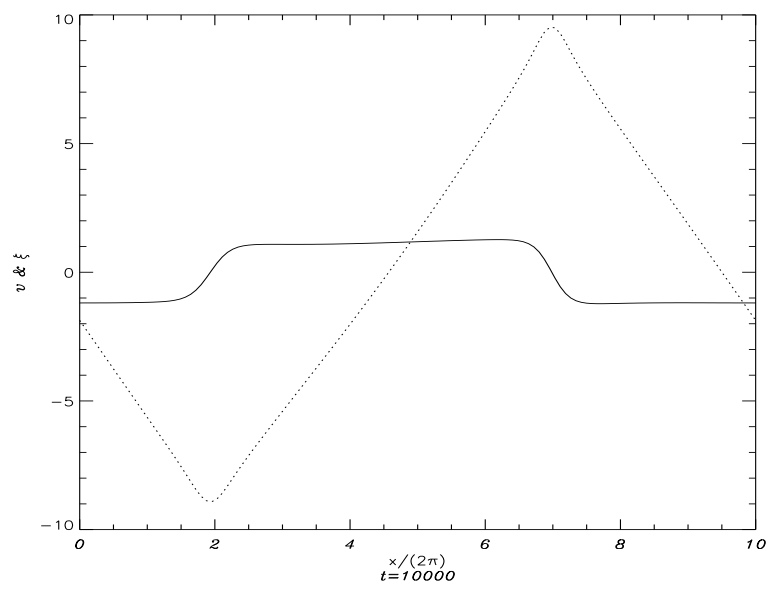

(b)

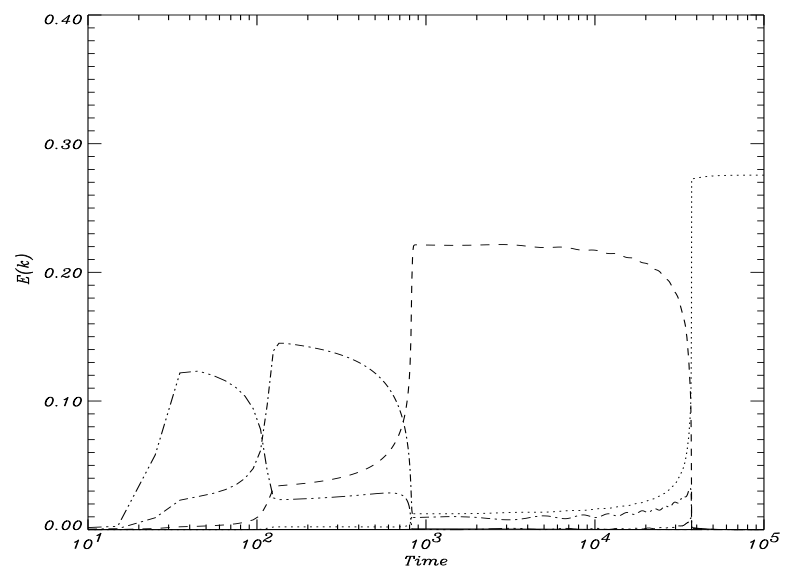

(d)

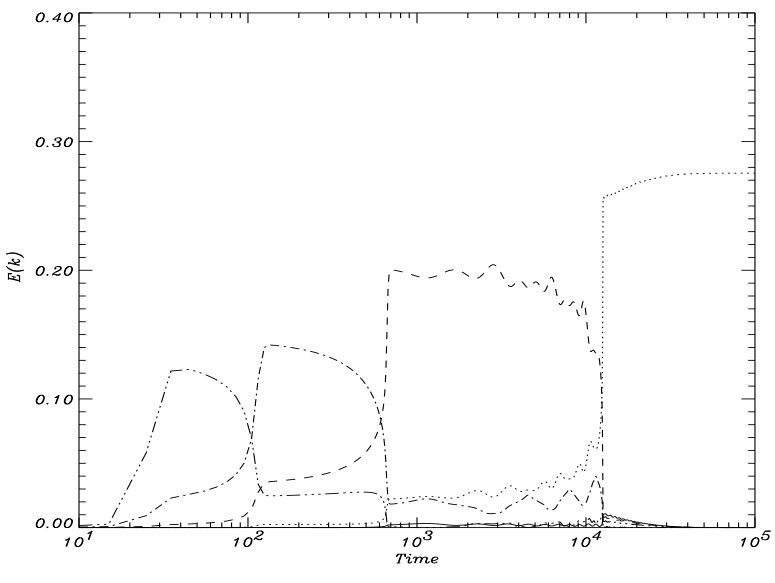

Figure 7: 
(a)
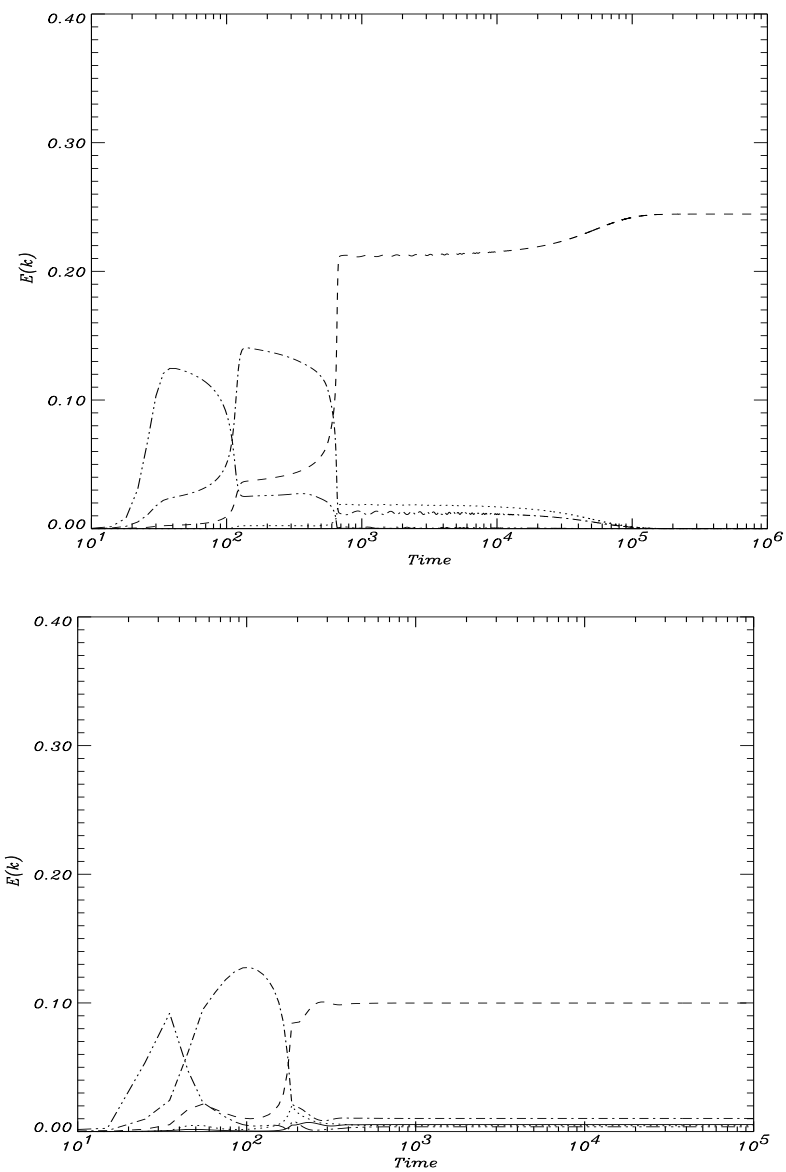

(b)

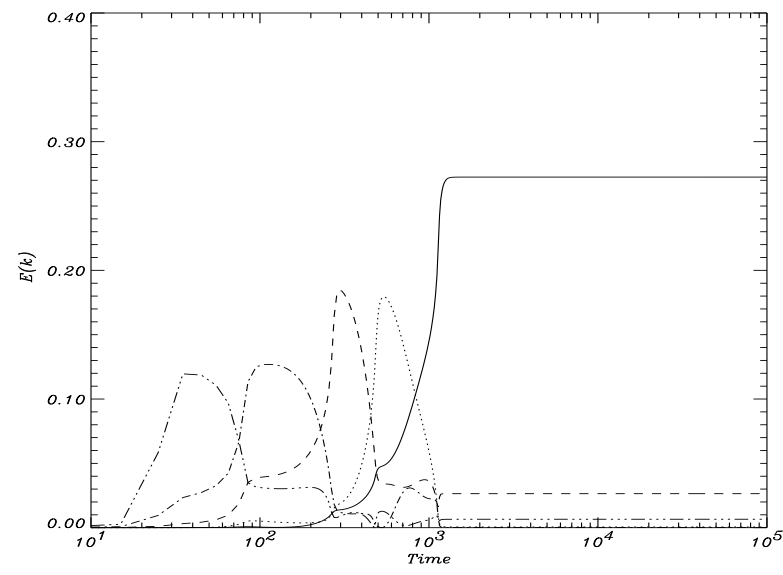

(d)

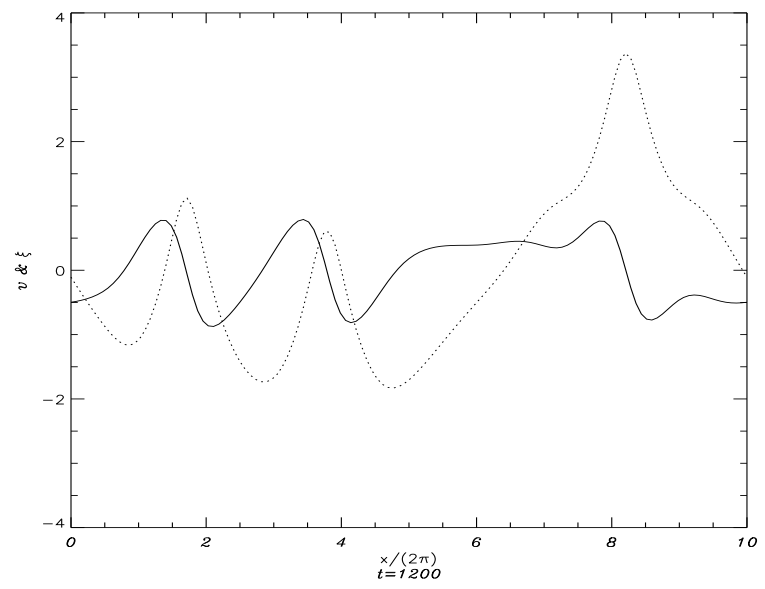

Figure 8: 
(a)

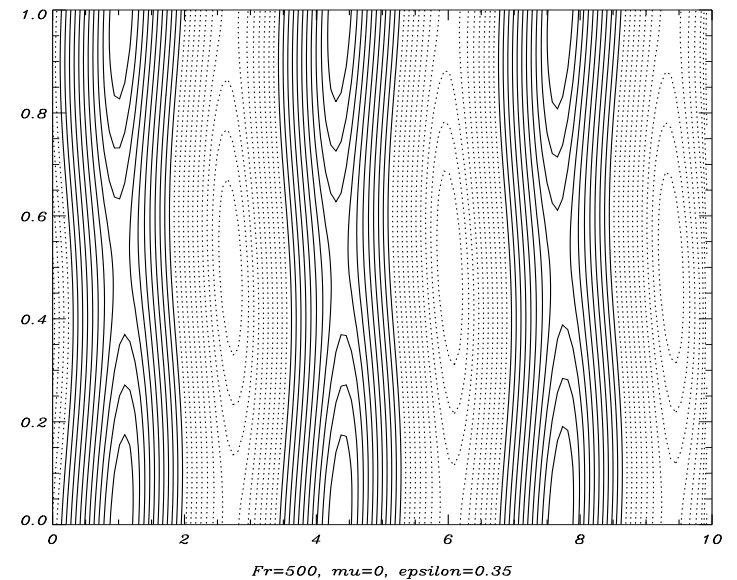

(c)

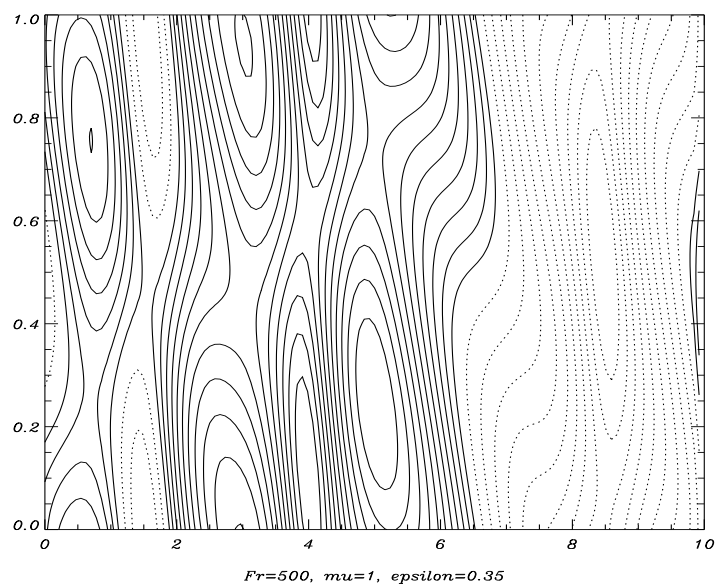

(b)

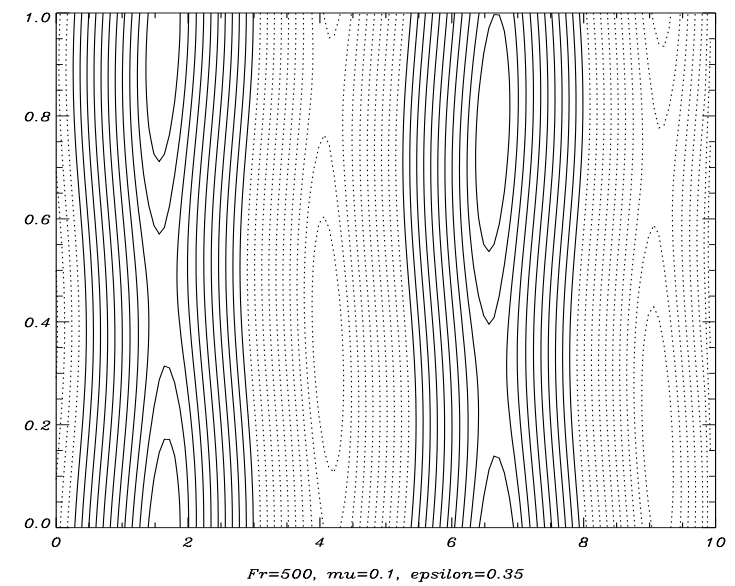

(d)

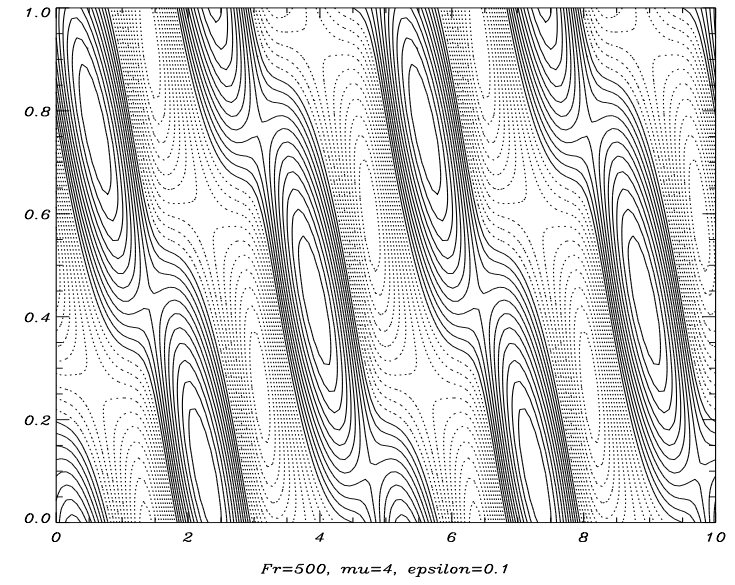

Figure 9:
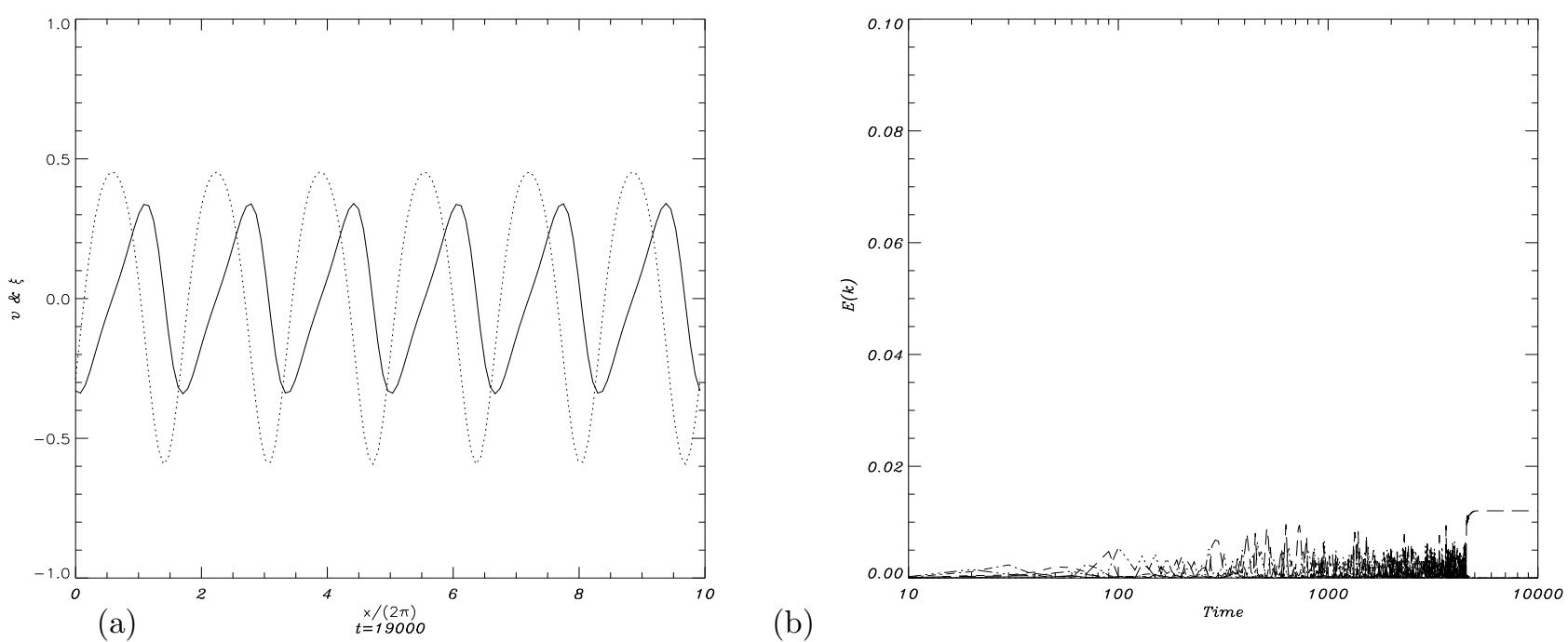

Figure 10: 